JOURNAL OF THE

AMERICAN MATHEMATICAL SOCIETY

Volume 16, Number 2, Pages 331-362

S 0894-0347(02)00416-2

Article electronically published on December 2, 2002

\title{
GENERAL ELEPHANTS OF THREE-FOLD DIVISORIAL CONTRACTIONS
}

\author{
MASAYUKI KAWAKITA
}

The theory of minimal models has enriched the study of higher-dimensional algebraic geometry; see [10] and [12. For a variety with mild singularities, this theory produces another variety which possesses good properties, after finite elementary transformations called divisorial contractions and flips. Since Mori completed this program in dimension three in [17, it has become desirable to study three-folds explicitly. This paper aims to complete the explicit study of three-fold divisorial contractions whose exceptional divisors contract to Gorenstein points, after the papers [7] and [8].

Reid pointed out that general elements in the anti-canonical systems of threefolds have at worst Du Val singularities in appropriate situations involving contractions of extremal faces. This has become known as the general elephant conjecture. The papers [19, 21] and 22] support it with affirmative answers for Fano threefolds with singularities, and this approach has settled the problem of the existence of three-fold flips in [11] and [17]. Our main theorem is that this conjecture holds for our divisorial contractions:

Theorem. Let $f:(Y \supset E) \rightarrow(X \ni P)$ be a germ of a three-fold divisorial contraction whose exceptional divisor $E$ contracts to a Gorenstein point $P$. Then a general element in the anti-canonical system $\left|-K_{Y}\right|$ has at worst Du Val singularities.

The statement is analogous to the flipping case, and we can start from a similar position after taking the intersection $C$ of $E$ and the birational transform of a general hyperplane section on $X$, because the first cohomology of $C$ vanishes. There is, however, a crucial difference between our curve $C$ and that in the flipping case. For any flipping curve in a three-fold, the first cohomology of any closed subscheme supported on this curve always vanishes, but this does not hold for our $C$. Nevertheless, provided that $P$ is Gorenstein, the numerical information on $f$ obtained in [7] allows us to analyse the local structure of $C \subset Y$ delicately. It encodes the behaviour of the global sections in $\left|-K_{Y}\right|$ and leads to our main theorem.

In fact, we prove more than the theorem in this paper. Let $S$ be a surface on $Y$ defined by a general element in $\left|-K_{Y}\right|$, and $S_{X}$ its birational transform on $X$. The theorem guarantees that $S$ and $S_{X}$ have only Du Val singularities, and the induced morphism $S \rightarrow S_{X}$ factors through the minimal resolution of $S_{X}$. We prove the theorem at the same time as providing information on the partial resolution $S \rightarrow S_{X}$; see Theorem 1.8. In some special cases, to be precise in types $\mathrm{O}$ and I in Theorem 1.4 the birational transform of a general hyperplane section on $X$ gives

Received by the editors October 22, 2001 and, in revised form, September 4, 2002.

2000 Mathematics Subject Classification. Primary 14E05, 14E30.

Key words and phrases. General elephant, divisorial contraction. 
a Du Val section $S$. On the other hand, in the remaining cases, we can obtain the strong version of the general elephant conjecture, which asserts that the type of any Du Val singularity $Q \in S$ is the same as that of a general Du Val section of a germ $Q \in Y$. In most cases this is proved by a direct search for $S$, but in some exceptional cases, where $-K_{Y}$ is linearly equivalent to the sum of $E$ and a Cartier divisor $L$, we obtain the desired $S$ by showing that $E$ has a Du Val singularity of the required type at any $Q \in S$, and that $L$ moves in a free linear system.

The theorem is applied to classify divisorial contractions. We describe the contractions to $\mathrm{c} A_{n}$ points in Theorem 1.13 following on from the smooth and the $\mathrm{c} A_{1}$ cases treated in 7] and [8. We expect that as the singularity at $P$ worsens, there are fewer divisorial contractions over $P$, because the choice of coordinates at $P$ is more restricted. However, since the defining equation of $X$ at $P$ becomes much more complicated, their explicit study is not necessarily simpler. Instead of complete descriptions in the remaining $\mathrm{c} D_{n}$ and $\mathrm{c} E_{n}$ cases, we give restrictions on the possible divisorial contractions by deriving an upper bound on discrepancies in Corollary 1.15. Now we have sufficient tools to classify divisorial contractions also in the $\mathrm{c} D_{n}$ and $\mathrm{c} E_{n}$ cases, once $P \in X$ has been given explicitly. What we should do is basically to compare discrepancies as in [9] or Lemma 6.1, using the enormous amount of information given by the singular Riemann-Roch technique and the general elephant theorem concerning the multiplicities along $E$ of surfaces inside $X$ with special directions. The connectedness lemma of Shokurov can be applied occasionally as in [3] Theorem 3.10] or [4, Theorem 3.6].

This paper is constructed as follows. In Section 1 we state our theorems precisely. Section 2 is devoted to preparing basic numerical techniques. After recalling the results for divisorial contractions to Gorenstein points in [7], we introduce the fundamental set-up to analyse $C \subset Y$, following [17]. The quite delicate local investigation of $C \subset Y$ is presented in Section 3 , Using this, in Section 4 we prove the main theorem, the existence of $\mathrm{Du}$ Val sections. In Section 5 we restrict the possible divisorial contractions, focusing on the types of singularities on Du Val sections. This provides an upper-bound on discrepancies in the $\mathrm{c} D_{n}$ and $\mathrm{c} E_{n}$ cases. Finally, in Section 6 we give an explicit description of divisorial contractions in the $\mathrm{c} A_{n}$ case.

\section{Statements}

We work over the complex number field $\mathbb{C}$.

Divisorial contractions play a major role in the minimal model program. This program was formulated to generalise the theory of minimal models of surfaces to higher-dimensional varieties. In order to avoid problems caused by the existence of small contractions, this program has to work in a category of varieties with mild singularities; see [10] and [12. Before we define a divisorial contraction, we have to introduce the class of terminal singularities. Let $X$ be a normal variety. We say that $X$ has at worst terminal singularities if $X$ is $\mathbb{Q}$-Gorenstein and if every exceptional divisor has positive coefficient in the discrepancy divisor $K_{Y}-f^{*} K_{X}$ for a resolution of singularities $f: Y \rightarrow X$.

The minimal model program works in the category of $\mathbb{Q}$-factorial normal varieties with terminal singularities; for such a variety, it produces a good variety after finite elementary transformations called divisorial contractions and flips. Since the main theorem is implied by the corresponding theorem in the analytic category, we define 
a divisorial contraction in a general sense. From now on, we work in the analytic category by relating to the algebraic category via the algebraisation theorems of Artin in [1] and [2].

Definition 1.1. Let $f: Y \rightarrow X$ be a proper morphism with connected fibres between normal varieties with at worst terminal singularities. We say that $f$ is a divisorial contraction if the exceptional locus of $f$ is a prime divisor and $-K_{Y}$ is $f$-ample.

Our study of three-fold divisorial contractions relies on the classification of threefold terminal singularities, which should be recalled. Let $P \in X$ be a three-fold germ. We say that $P$ is a $c D V$ (compound $D u V a l$ ) point if a general hyperplane section has at worst a Du Val singularity at $P$. The singularity $P$ is said to be $c A_{n}$, $c D_{n}, c E_{n}$ (compound $A_{n}, D_{n}, E_{n}$ ) according to the type of the Du Val singularity on a general hyperplane section. For convenience, we say that a smooth point on a surface or a three-fold is $A_{0}$, respectively c $A_{0}$. It is a result of Reid that three-fold Gorenstein terminal singularities are characterised as isolated cDV points:

Theorem 1.2 ([18, Theorem1.1]). Let $P \in X$ be a three-fold germ. $P$ is a Gorenstein terminal singularity if and only if $P$ is an isolated $c D V$ point.

We then consider a three-fold non-Gorenstein terminal singularity $P \in X$. Let $r$ be the local Gorenstein index of $P \in X$, that is, the smallest positive integer such that $r K_{X}$ is Cartier at $P$. Take the index-one cover $\pi:\left(X^{\sharp} \ni P^{\sharp}\right) \rightarrow(X \ni P)$, which is a cyclic $\mu_{r}$-cover. Fix a character generating $\operatorname{Hom}\left(\mu_{r}, \mathbb{C}^{\times}\right)=\mathbb{Z} /(r)$ and define the weight modulo $r$ for any semi-invariant function on $X^{\sharp}$ with respect to this character.

Theorem 1.3 (16]). There exists a $\mu_{r}$-equivariant identification

$$
P^{\sharp} \in X^{\sharp} \cong o \in(\phi=0) \subset \mathbb{C}_{x_{1} x_{2} x_{3} x_{4}}^{4},
$$

where $x_{1}, x_{2}, x_{3}, x_{4}$ and $\phi$ are $\mu_{r}$-semi-invariant, and $\phi=x_{4}$ if $P^{\sharp} \in X^{\sharp}$ is a smooth point. The weights of $x_{1}, x_{2}, x_{3}, x_{4}$ and $\phi$ satisfy one of the following:

(i) $\operatorname{wt}\left(x_{1}, x_{2}, x_{3}, x_{4} ; \phi\right)=(1,-1, b, 0 ; 0)$, where $b$ is co-prime to $r$.

(ii) $r=4$ and $\operatorname{wt}\left(x_{1}, x_{2}, x_{3}, x_{4} ; \phi\right)=(1,3,3,2 ; 2)$.

Mori gave more precise description of $\phi$ and weights in [16], and the classification is completed by [13, Theorem 6.4].

According to the above classification, any three-fold terminal singularity $P \in X$ has a small deformation to a basket of terminal quotient singularities $P_{i}$. The $P_{i}$ are called fictitious singularities in the sense of Reid in [20]. Note that if $P$ is of type (i) in Theorem 1.3. any local index at $P_{i}$ equals that at $P$, and if $P$ is of type (iii), one of $P_{i}$ has local index 2 and all the others have local index 4.

We return to the study of three-fold divisorial contractions. Let

$$
f:(Y \supset E) \rightarrow(X \ni P)
$$

be a germ of a three-fold divisorial contraction whose exceptional divisor $E$ contracts to a point $P$. There are two ways to study $f$, namely, one starting from $X$, and the other from $Y$. By the former approach, Mori classified them in the case where $Y$ is smooth in [15, and Cutkosky extended this result to the case where $Y$ is Gorenstein in [5]. On the other hand, by the latter approach, Kawamata proved that $f$ is a certain weighted blow-up when $P$ is a terminal quotient singularity in 
9], and Corti proved that $f$ is the blow-up when $P$ is an ordinary double point in [3, Theorem 3.10].

Now is the time that we state our theorems. We start with a numerical classification of $f$. From now on, we assume that $P$ is a Gorenstein point. As in Section 2 set $K_{Y}=f^{*} K_{X}+a E$, and let $I:=\left\{Q:\right.$ type $\left.\frac{1}{r_{Q}}\left(1,-1, \overline{a v_{Q}}\right)\right\}$ with $v_{Q} \leq r_{Q} / 2$ be the basket of fictitious singularities from non-Gorenstein singularities on $Y$. We also set $J:=\left\{\left(r_{Q}, v_{Q}\right)\right\}_{Q \in I}$ and $1+d(-1):=\operatorname{dim} \mathcal{O}_{X} / f_{*} \mathcal{O}_{Y}(-2 E)$. The following theorem is the same as [7. Theorem 4.5], except for the statement that $d(-1) \neq 0$ in type $\mathrm{O}$. This additional statement is due to Theorem 2.7.

Theorem 1.4. $f$ belongs to exactly one of the types in the following table. If $f$ is of type $I V$, then $f$ is the usual blow-up along a smooth point $P$.

\begin{tabular}{l|c|l|l}
\hline type & $1+d(-1)$ & \multicolumn{1}{|c|}{$J$} & \multicolumn{1}{|c}{$a$} \\
\hline$O$ & $\geq 2$ & & 1 \\
$I$ & 1 & $\{(7,3)\}$ or $\{(3,1),(5,2)\}$ & 2 \\
$I I a$ & 2 & $\{(r, 2)\}$ & $4 / r E^{3}=2$ or 4 \\
$I I b$ & 2 & $\left\{\left(r_{1}, 1\right),\left(r_{2}, 1\right)\right\}$ & $\left(r_{1}+r_{2}\right) / r_{1} r_{2} E^{3} \geq 2$ \\
$I I I$ & 3 & $\{(r, 1)\}$ & $(1+r) / r E^{3} \geq 2$ \\
$I V$ & 4 & $\emptyset$ & 2 \\
\hline
\end{tabular}

For convenience, we divide type IIb into two types according to the number of non-Gorenstein points on $Y$. We say that $f$ is of type $\mathrm{IIb}^{\vee}$ or $\mathrm{IIb}^{\vee \vee}$ if $f$ is of type II with one, respectively two non-Gorenstein points on $Y$.

Remark 1.5. If $f$ is of type $\mathrm{IIb}^{\vee}$, then $J=\{(r, 1),(r, 1)\}$ or $\{(2,1),(4,1)\}$, and $a=2$, respectively 3. By [16], the unique non-Gorenstein point $Q \in Y$ can be described as one of the following equations $\phi=0$, using semi-invariant local coordinates $y_{1}, y_{2}, y_{3}, y_{4}$ of the index-one cover $Q^{\sharp} \in Y^{\sharp}$ with weights $w=\operatorname{wt}\left(y_{1}, y_{2}, y_{3}, y_{4}\right)$.

(i) $J=\{(r, 1),(r, 1)\}$.

(a) $\phi=y_{1} y_{2}+g\left(y_{3}^{r}, y_{4}\right)$ and $w=(1,-1,2,0) \cdot g\left(0, y_{4}\right)$ has order 2 . The general Du Val section of a germ $Q \in Y$ is $A_{2 r-1}$.

(b) $r=3, \phi=y_{4}^{2}+\phi_{3}\left(y_{1}, y_{2}, y_{3}\right)+\phi_{\geq 4}\left(y_{1}, y_{2}, y_{3}\right)$ and $w=(1,-1,2,0)$. $\phi_{3}$ is $y_{1}^{3}+y_{2}^{3}+y_{3}^{3}, y_{1}^{3}+y_{2} y_{3}^{2}$, or $y_{1}^{3}+y_{2}^{3}$, and $\phi_{\geq 4}$ has order $\geq 4$. The general Du Val section of a germ $Q \in Y$ is $E_{6}$.

(ii) $J=\{(2,1),(4,1)\} . \phi=y_{1}^{2}+y_{2}^{2}+g\left(y_{3}^{2}, y_{4}\right)$ and $w=(1,3,3,2) . g\left(0, y_{4}\right)$ has order 3. The general Du Val section of a germ $Q \in Y$ is $D_{5}$.

The following definition is due to Reid:

Definition $1.6([20])$. The general elements in the anti-canonical system are called general elephants.

In 20] Reid proposed the general elephant conjecture. It is that general elephants of three-folds should have at worst Du Val singularities in appropriate situations involving the contractions of extremal faces. Our main theorem is that this conjecture holds for our divisorial contractions:

Theorem 1.7. A general elephant of $Y$ has at worst Du Val singularities. 
Let $S$ be a general elephant of $Y$, and $S_{X}$ its birational transform on $X$. By Theorem 1.7. $S$ and $S_{X}$ have at worst Du Val singularities and the induced morphism $S \rightarrow S_{X}$ factors through the minimal resolution of $S_{X}$. We obtain Theorem 1.7 at the same time as providing the following information on the partial resolution $S \rightarrow S_{X}$. Theorems 1.7 and 1.8 are implied by Theorems $2.7,4.2$ and 4.4 and the results in Section 5 .

Theorem 1.8. $\quad$ (i) Assume that $f$ is of type $O$ or $I$. Then $S_{X}$ is a general elephant of $X$. Moreover, if $f$ is of type $I$, then $P$ is $c E_{7}$ or $c E_{8}$.

(ii) Assume that $f$ is of type II, III or IV. Then possible types of Du Val singularities on $S$ and $S_{X}$ are as in the following table. The type of any $D u$ Val singularity $Q \in S$ is the same as that of a general elephant of a germ $Q \in Y$. Moreover, the partial resolution $S \rightarrow S_{X}$ has irreducible exceptional locus except in the case IIa when $S_{X}$ is of the alternative type $D_{r+1}$, or in the case $I I b^{\vee}$ in the second line of the table with $S_{X}$ of type $D_{2 r+1}$.

\begin{tabular}{l|l|l}
\hline \multicolumn{1}{c|}{ type of $f$} & \multicolumn{1}{|c}{ type of $S_{X}$} & \multicolumn{1}{c}{ type of $S$} \\
\hline$I I a$ & $D_{r}$ or $D_{r+1}$ & $A_{r-1}$ \\
$I I b^{\vee}, J=\{(r, 1),(r, 1)\}$ & $D_{2 r}$ or $D_{2 r+1}$ & $A_{2 r-1}$ \\
$I I b^{\vee}, J=\{(3,1),(3,1)\}$ & $E_{7}$ & $E_{6}$ \\
$I I b^{\vee}, J=\{(2,1),(4,1)\}$ & $E_{6}$ & $D_{5}$ \\
$I I b^{\vee \vee}$ & $A_{r_{1}+r_{2}-1}$ & $A_{r_{1}-1}$ and $A_{r_{2}-1}$ \\
$I I I$ & $A_{r}$ & $A_{r-1}$ \\
$I V$ & smooth & smooth \\
\hline
\end{tabular}

We have examples of type I with $J=\{(3,1),(5,2)\}$ in Example 5.3 but I do not know whether type I with $J=\{(7,3)\}$ happens or not. If $f$ is of type I with $J=\{(7,3)\}$, then $P$ has to be $\mathrm{c} E_{7}$ by Remark 5.2

The main theorem can be applied to classify divisorial contraction. We classify them according the type of $P$. Divisorial contractions to smooth or $\mathrm{c} A_{1}$ points are completely described in the papers [7] and [8]:

Theorem 1.9 (7]). Assume that $P$ is smooth. Then $f$ is a weighted blow-up. More precisely, we can take local coordinates $x_{1}, x_{2}, x_{3}$ at $P$ and co-prime positive integers $s$ and $t$, such that $f$ is the weighted blow-up of $X$ with weights $\operatorname{wt}\left(x_{1}, x_{2}, x_{3}\right)=$ $(1, s, t)$. Moreover, any such $f$ is a divisorial contraction.

Remark 1.10. We may assume that $s \leq t$. The type of $f$ is $\mathrm{IIb}^{\vee \vee}$ if $s>1$, III if $s=1$ and $t>1$, and IV if $t=1$.

Theorem $1.11([8])$. Assume that $P$ is $c A_{1}$. Then $f$ is a weighted blow-up. More precisely, under a suitable identification

$$
P \in X \cong o \in\left(x_{1} x_{2}+x_{3}^{2}+x_{4}^{N}=0\right) \subset \mathbb{C}_{x_{1} x_{2} x_{3} x_{4}}^{4},
$$

$f$ is the weighted blow-up with one of the following weights:

(i) $\operatorname{wt}\left(x_{1}, x_{2}, x_{3}, x_{4}\right)=(s, 2 t-s, t, 1)$, where $s, t$ are co-prime positive integers such that $s \leq t \leq N / 2$.

(ii) $N=3$ and $\operatorname{wt}\left(x_{1}, x_{2}, x_{3}, x_{4}\right)=(1,5,3,2)$.

Moreover, any such $f$ is a divisorial contraction. 
Remark 1.12. In (i), the type of $f$ is $\mathrm{IIb}^{\vee \vee}$ if $s>1$, III if $s=1$ and $t>1$, and O if $t=1$. In (iii), the type of $f$ is IIa.

In Section 6 of this paper, we describe divisorial contractions to $\mathrm{c} A_{n}$ points with $n \geq 2$ :

Theorem 1.13. Assume that $P$ is $c A_{n}$ for some $n \geq 2$. Then one of the following holds:

(i) Under a suitable identification

$$
P \in X \cong o \in\left(x_{1} x_{2}+g\left(x_{3}, x_{4}\right)=0\right) \subset \mathbb{C}_{x_{1} x_{2} x_{3} x_{4}}^{4},
$$

$f$ is the weighted blow-up with weights $\mathrm{wt}\left(x_{1}, x_{2}, x_{3}, x_{4}\right)=\left(r_{1}, r_{2}, a, 1\right)$, where a divides $r_{1}+r_{2}$ and is co-prime to $r_{1}$ and $r_{2}, g$ has weighted order $r_{1}+r_{2}$ with weights $\operatorname{wt}\left(x_{3}, x_{4}\right)=(a, 1)$, and the monomial $x_{3}^{\left(r_{1}+r_{2}\right) / a}$ appears in $g$ with non-zero coefficient. Moreover, any such $f$ is a divisorial contraction.

(ii) $\mathrm{P}$ is a $c A_{2}$ point isomorphic to

$$
o \in\left(x_{1} x_{2}+x_{3}^{3}+g_{\geq 4}\left(x_{3}, x_{4}\right)=0\right) \subset \mathbb{C}_{x_{1} x_{2} x_{3} x_{4}}^{4},
$$

where $g_{\geq 4}$ has total order $\geq 4$ in $x_{3}, x_{4}, Y$ has exactly one non-Gorenstein point $Q$, isomorphic to o $\in\left(y_{1}^{2}+y_{2}^{2}+y_{3}^{2}+y_{4}^{3}=0\right)$ in the quotient space $\mathbb{C}_{y_{1} y_{2} y_{3} y_{4}}^{4} / \frac{1}{4}(1,3,3,2)$, and $K_{Y}=f^{*} K_{X}+3 E$. Moreover, such an example exists in Example 6.8.

Remark 1.14. In (1), we may assume that $r_{1} \leq r_{2}$. The type of $f$ is IIb ${ }^{\vee \vee}$ if $r_{1}>1$ and $a>1$, III if $r_{1}=1$ and $a>1$, and O if $a=1$. In (iii), the type of $f$ is IIb $^{\vee}$ with $J=\{(2,1),(4,1)\}$.

We note that Corti obtained the result in the case where $P$ is an ordinary double point in [3, Theorem 3.10], and that Corti and Mella obtained the result in the case where $P$ is a $\mathrm{c} A_{2}$ point isomorphic to $o \in\left(x_{1} x_{2}+x_{3}^{3}+x_{4}^{3}=0\right) \subset \mathbb{C}^{4}$ in [4, Theorem 3.6].

On the other hand, in the remaining $\mathrm{c} D_{n}$ and $c E_{n}$ cases we derive an upperbound on the discrepancy $a$. The next corollary follows straightforwardly from Theorem 1.8:

Corollary 1.15. Assume that $P$ is $c D_{n}$ or $c E_{n}$. Then $f$ is of type O, I, IIa or $I I b^{\vee}$ in Theorem 1.4, and the discrepancy $a \leq 4$.

We have examples of type $\mathrm{O}, \mathrm{I}, \mathrm{IIb}^{\vee}$, where $a=1,2,3$, in Examples $2.3,5.35 .4$ and 5.5. However I do not know whether type IIa happens or not. If $a=4$ in the $\mathrm{c} D_{n}$ or $\mathrm{c} E_{n}$ case, then $r=5$ by Theorem 3.5)(iii) and $P$ has to be $\mathrm{c} D_{4}, \mathrm{c} D_{5}$ or $\mathrm{c} D_{6}$.

\section{BASIC NUMERICAL RESUltS}

Let $f:(Y \supset E) \rightarrow(X \ni P)$ be a germ of a three-fold divisorial contraction whose exceptional divisor $E$ contracts to a Gorenstein point $P$. Remark that $f$ can be always extended to a morphism between projective varieties by the algebraisation theorem in [1] and [2].

Set $K_{Y}=f^{*} K_{X}+a E$, and let $r$ be the global Gorenstein index of $Y$. Note that $a$ and $r$ are co-prime by [7, Lemma 4.3]. We take an integer $e$ such that $a e \equiv 1$ modulo $r$. Let $I:=\left\{Q\right.$ : type $\left.\frac{1}{r_{Q}}\left(1,-1, b_{Q}\right)\right\}$ be the basket of fictitious singularities from non-Gorenstein singularities on $Y$. Then $\left(\mathcal{O}_{Y_{Q}}\left(E_{Q}\right)\right)_{Q} \cong\left(\mathcal{O}_{Y_{Q}}\left(e K_{Y_{Q}}\right)\right)_{Q}$, where 
$\left(Y_{Q}, E_{Q}\right)$ is the deformed pair at $Q$ from $(Y, E)$. We note that $b_{Q}$ is co-prime to $r_{Q}$ and that $e$ is also co-prime to $r_{Q}$ since $a e \equiv 1$ modulo $r$. Hence $v_{Q}:=\overline{e b_{Q}}$ is co-prime to $r_{Q}$. Here ${ }^{-}$denotes the smallest residue modulo $r_{Q}$, that is, $\bar{j}:=j-\left\lfloor\frac{j}{r_{Q}}\right\rfloor r_{Q}$, where \lfloor\rfloor denotes the round-down, that is, $\lfloor j\rfloor:=\max \{k \in \mathbb{Z} \mid k \leq j\}$. Replacing $b_{Q}$ with $r_{Q}-b_{Q}$ if necessary, we may assume that $v_{Q} \leq r_{Q} / 2$. With this description, $r=1$ if $I$ is empty, and otherwise $r$ is the lowest common multiple of $\left\{r_{Q}\right\}_{Q \in I}$. Set $J:=\left\{\left(r_{Q}, v_{Q}\right)\right\}_{Q \in I}$. We moreover define $d(i):=\operatorname{dim} f_{*} \mathcal{O}_{Y}(i E) / f_{*} \mathcal{O}_{Y}((i-1) E)$. Note that $d(i)=0$ for $i \geq 1$ and $d(0)=1$.

Remark 2.1. If $P$ is a singular point, then $a<\max \left\{r_{Q}\right\}$ unless $a \neq 1$. This is by an analogue to the proof of [8, Lemma 6.10], because there exists a valuation with centre $P$ the discrepancy of which with respect to $K_{X}$ is 1 by [14.

We have a rough numerical classification of $f$ by 7 Theorem 4.5], which is the same as Theorem 1.4 except for the statement that $d(-1) \neq 0$ in type O. We quote this classification by the label [7, Theorem 4.5] until the additional statement is deduced from Theorem 2.7 .

Remark 2.2. The possible values of $J$ can be restricted even if $f$ is of type $O$, according to the proof of [7, Theorem 4.5]. In fact, $J$ is one of

$\{(7,3)\},\{(8,3)\},\{(2,1),(5,2)\},\{(3,1),(5,2)\},\{(4,1),(5,2)\},\{(2,1),(7,2)\}$,

$\left\{(2,1),(2,1),\left(r_{3}, 1\right)\right\},\{(2,1),(3,1),(3,1)\},\{(2,1),(3,1),(4,1)\}$,

$\{(2,1),(3,1),(5,1)\},\{(r, 2)\},\left\{\left(r_{1}, 1\right),\left(r_{2}, 1\right)\right\},\{(r, 1)\}$ and $\emptyset$.

In particular, the number of the fictitious singularities is $\leq 3$.

Example 2.3. The weighted blow-up of the $\mathrm{c} D_{4}$ singularity $o \in\left(x_{1}^{2}+x_{2}^{3}+x_{3}^{3}+\right.$ $\left.x_{4}^{6}=0\right) \subset \mathbb{C}^{4}$ with weights wt $\left(x_{1}, x_{2}, x_{3}, x_{4}\right)=(3,2,2,1)$ in [7. Example 4.6] is an example for which $J$ is $\{(2,1),(2,1),(2,1)\}$.

The numerical classification comes from the singular Riemann-Roch formula [20] Theorem 10.2] and a relative vanishing theorem [10, Theorem 1-2-5] with the exact sequences

$$
0 \rightarrow \mathcal{O}_{Y}((i-1) E) \rightarrow \mathcal{O}_{Y}(i E) \rightarrow \mathcal{Q}_{i} \rightarrow 0 .
$$

The sheaves $\mathcal{Q}_{i}$ are $\mathrm{S}_{2}$ by [12, Proposition 5.26], and are reflexive since they are locally free on the restriction $E^{o} \subseteq E$ of the Gorenstein locus of $Y$. Thus, since $E$ is Cohen-Macaulay, we have

$$
\mathcal{Q}_{i}=i_{*}\left(\left.\mathcal{O}_{Y}(i E) \otimes \mathcal{O}_{E}\right|_{E^{o}}\right),
$$

where $i$ is the induced map $E^{o} \hookrightarrow E$.

The singular Riemann-Roch formula implies

$$
\begin{aligned}
\chi\left(\mathcal{Q}_{i}\right)= & \frac{1}{12}\left\{2\left(3 i^{2}-3 i+1\right)-3(2 i-1) a+a^{2}\right\} E^{3} \\
& +\frac{1}{12} E \cdot c_{2}(Y)+A_{i}-A_{i-1}, \\
A_{i}:= & \sum_{Q \in I}\left(-\overline{i e} \frac{r_{Q}^{2}-1}{12 r_{Q}}+\sum_{j=1}^{\overline{i e}-1} \frac{\overline{j b_{Q}}\left(r_{Q}-\overline{j b_{Q}}\right)}{2 r_{Q}}\right) .
\end{aligned}
$$

On the other hand a relative vanishing theorem implies

$$
R^{j} f_{*} \mathcal{O}_{Y}(i E)=0 \quad(i \leq a, j \geq 1) .
$$

We summarise the formulae in [7] Section 4] obtained from the above equalities: 
Proposition 2.4. $\quad$ (i) $r E^{3} \in \mathbb{Z}_{>0}$.

(ii) $d(i)=\chi\left(\mathcal{Q}_{i}\right)$ for $i \leq a$.

(iii) If $f$ is of type IIb or III and we set $r_{1}=1, r_{2}=r$ if $f$ is of type III, then

$d(-i)=1+\left\lfloor\frac{i}{r_{1}}\right\rfloor \quad\left(0 \leq i<\min \left\{r_{2}, a\right\}\right)$.

(iv) Set $B_{i}:=\sum_{Q \in I} \frac{\overline{i v_{Q}}\left(r_{Q}-\overline{i v_{Q}}\right)}{2 r_{Q}}$. Then

$$
\chi\left(\mathcal{Q}_{-i}\right)-\chi\left(\mathcal{Q}_{i+1}\right)=\left(i+\frac{1}{2}\right) a E^{3}+B_{i+1}-B_{i} .
$$

Remark 2.5. The values of $d(-i)=\chi\left(\mathcal{Q}_{-i}\right)$ below are used later:

(i) (type IIa) By direct calculation using (2.2), we obtain $d(-1)=1$ and $d(-2) \geq 2$ if $a=2$ or $(a, r)=(4,5)$.

(ii) (types IIb and III) $d(-i)=1+\left\lfloor\frac{i}{r_{1}}\right\rfloor$ for $1 \leq i<\min \left\{r_{2}, a\right\}$ by Proposition 2.4 (iii), and $d(-a)=2+\left\lfloor\frac{a}{r_{1}}\right\rfloor$ if $a<r_{2}$ by Lemma 2.6 and the proofs of [7. Proposition 4.4.3] and the latter part of [7, Theorem 4.5].

Lemma 2.6. $\mathcal{Q}_{a+1}=\omega_{E}$ and $h^{0}\left(\omega_{E}\right)=h^{1}\left(\omega_{E}\right)=0, h^{2}\left(\omega_{E}\right)=1$.

Proof. Let $E^{o}$ on $E$ be the restriction of the Gorenstein locus on $Y$, and $i: E^{o} \hookrightarrow E$ the induced map. $\omega_{E}=i_{*} \omega_{E^{o}}$ since $\omega_{E}$ is $\mathrm{S}_{2}$. Thus $\mathcal{Q}_{a+1}=\omega_{E}$ by $\left.\mathcal{Q}_{a+1}\right|_{E^{o}}=\omega_{E^{o}}$. $H^{i}\left(\omega_{E}\right)$ is the dual of $\operatorname{Ext}_{E}^{2-i}\left(\omega_{E}, \omega_{E}\right)$. Thus $h^{0}\left(\omega_{E}\right)=h^{1}\left(\omega_{E}\right)=0$ since $E$ is Cohen-Macaulay, and $h^{2}\left(\omega_{E}\right)=\operatorname{dim} \operatorname{Hom}\left(\omega_{E}, \omega_{E}\right)$. The map $\operatorname{Hom}\left(\omega_{E}, \omega_{E}\right) \rightarrow$ $\operatorname{Hom}\left(\omega_{E^{o}}, \omega_{E^{o}}\right)$ is injective since $\omega_{E}$ is $\mathrm{S}_{2}$, and $\operatorname{dim} \operatorname{Hom}\left(\omega_{E^{o}}, \omega_{E^{o}}\right)=h^{0}\left(\mathcal{O}_{E^{o}}\right)=1$. Hence $h^{2}\left(\omega_{E}\right)=1$ by $\operatorname{Hom}\left(\omega_{E}, \omega_{E}\right) \neq 0$.

Let $P \in H_{X}$ be a general hyperplane section on $X$, and $H$ its birational transform on $Y$. Set $f^{*} H_{X}=H+b E$. Then $b$ is the largest integer for which $f_{*} \mathcal{O}_{Y}(-b E)=$ $\mathfrak{m}_{P}$. Let $Y^{o}$ be the Gorenstein locus of $Y$. By the adjunction formula, we have

$$
\left.\omega_{H}\right|_{Y^{\circ} \cap H}=\left.f^{*} \omega_{H_{X}} \otimes \mathcal{O}_{Y}((a-b) E)\right|_{Y^{\circ} \cap H} .
$$

$a \geq b$ because $H_{X}$ is canonical. Moreover, if $a=b$, then $H$ is normal and gives a $\mathrm{Du}$ Val section in the anti-canonical system of $Y$. Combining it with 7, Theorem 4.5], we obtain the following theorem:

Theorem 2.7. If $f$ is of type $O$ or $I$, then the birational transform $H$ of a general hyperplane section $H_{X}$ on $X$ gives a Du Val section in the anti-canonical system of $Y$.

We investigate the scheme $H \cap E$ in the remainder of this section. We use the following commutative diagrams repeatedly:

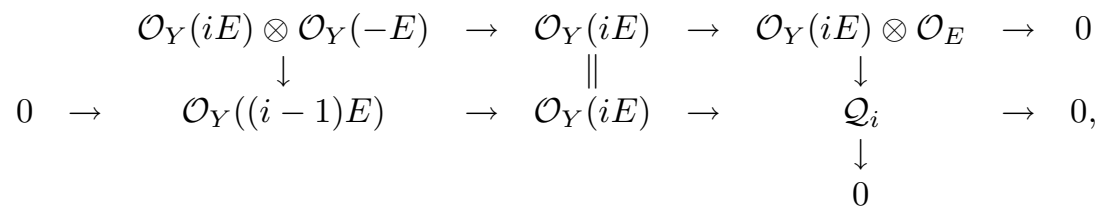




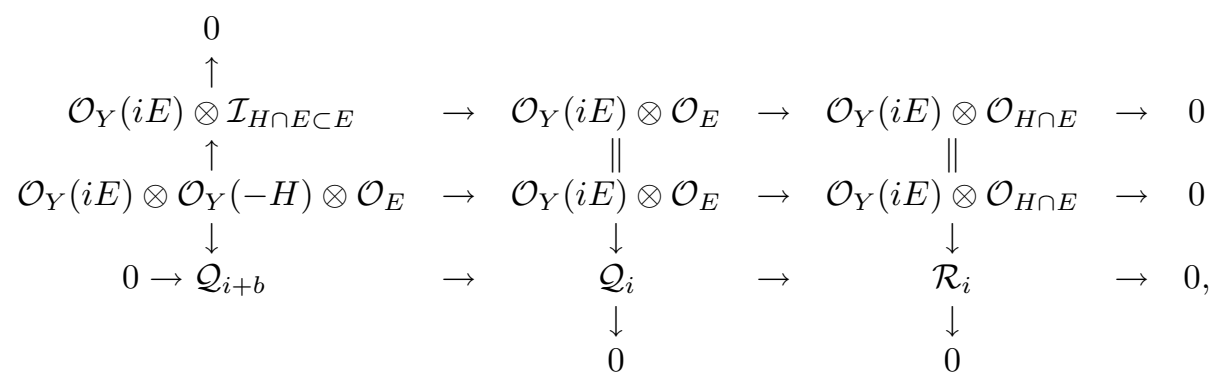

where $b$ is defined as above. Chasing these diagrams, we have the following equalities by (2.3) and Lemma 2.6.

Lemma 2.8. $\quad$ (i) $h^{j}\left(\mathcal{O}_{Y}(i E) \otimes \mathcal{O}_{E}\right)=0$ for $i \leq a, j \geq 1$, and $h^{1}\left(\mathcal{O}_{Y}((a+1) E) \otimes \mathcal{O}_{E}\right)=0, h^{2}\left(\mathcal{O}_{Y}((a+1) E) \otimes \mathcal{O}_{E}\right)=1$.

(ii) If $f$ is not of type $I$, then $h^{1}\left(\mathcal{O}_{Y}(i E) \otimes \mathcal{O}_{H \cap E}\right)=0$ for $i \leq a-1$, and $h^{1}\left(\mathcal{O}_{Y}(a E) \otimes \mathcal{O}_{H \cap E}\right)=1$.

Remark 2.9. $h^{0}\left(\mathcal{O}_{H \cap E}\right)=1$ and $h^{1}\left(\mathcal{O}_{H \cap E}\right)=0$ in any case, even if $f$ is of type I by Theorem 2.7. In particular, $H \cap E$ has no embedded points and $(H \cap E)_{\text {red }}$ is a union of $\mathbb{P}^{1}$.

The main ingredient we study is an irreducible reduced subscheme $C \cong \mathbb{P}^{1} \subseteq$ $H \cap E$. Here we introduce a normal form of $Q \in C \subset Y$ at each non-Gorenstein point $Q$ of $Y$ through which $C$ passes, following [17] Lemma 2.7]. Let $r_{Q}$ be the local index of $Q \in Y$. Take the index-one cover $\pi:\left(Y^{\sharp} \ni Q^{\sharp}\right) \rightarrow(Y \ni Q)$ and set $C^{\sharp}:=\left(C \times_{Y} Y^{\sharp}\right)_{\text {red }}$. We have an identification in Theorem 1.3.

$$
Q^{\sharp} \in Y^{\sharp} \cong o \in(\phi=0) \subset \mathbb{C}_{x_{1} x_{2} x_{3} x_{4}}^{4} .
$$

Let $s_{Q}$ be the number of the irreducible components of $C^{\sharp}$, and $Q^{\dagger} \in C^{\dagger}$ the normalisation of one of the irreducible components of $C^{\sharp}$. Let $t \in \mathcal{O}_{C, Q}$ and $t^{s_{Q} / r_{Q}} \in$ $\mathcal{O}_{C^{\dagger}, Q^{\dagger}}$ be uniformising parameters of $C$ and $C^{\dagger}$. Let $a_{i}$ be the minimal number such that there exists a semi-invariant function with weight wt $x_{i}$ whose image in $\mathcal{O}_{C^{\dagger}, Q^{\dagger}}$ has order $a_{i} / r_{Q}$ with respect to $t$. Note that

$$
\left(a_{1}, a_{2}, a_{3}, a_{4}\right) \in s_{Q} \mathbb{Z}\left(\text { wt } x_{1}, \text { wt } x_{2}, \text { wt } x_{3}, \text { wt } x_{4}\right) \subseteq\left(\mathbb{Z} /\left(r_{Q}\right)\right)^{4} .
$$

Then we can take an identification in Theorem 1.3 such that $\left.x_{i}\right|_{C^{\dagger}}=t^{a_{i} / r_{Q}}$ for $i=1,2,3$, and $\left.x_{4}\right|_{C^{\dagger}}=t^{a_{4} / r_{Q}}$ if $Q^{\sharp} \in Y^{\sharp}$ is singular.

We show how to compute the images of some natural maps of sheaves defined on a germ $Q \in C \subset Y$ using the above data $a_{1}, a_{2}, a_{3}, a_{4}$. For $n \in \mathbb{Z} /\left(r_{Q}\right)$, write $w_{Q}^{C}(n)$ for the smallest non-negative integer such that $\left(w_{Q}^{C}(n), n\right) \in \mathbb{Z} \times \mathbb{Z} /\left(r_{Q}\right)$ is contained in the semi-group

$$
\begin{aligned}
\mathbb{Z}_{\geq 0}\left(a_{1}, \text { wt } x_{1}\right)+\mathbb{Z}_{\geq 0}\left(a_{2}, \text { wt } x_{2}\right)+\mathbb{Z}_{\geq 0}\left(a_{3}, \text { wt } x_{3}\right)+\mathbb{Z}_{\geq 0}\left(a_{4}, \text { wt } x_{4}\right), & \text { or } \mathbb{Z}_{\geq 0}\left(a_{1}, \text { wt } x_{1}\right)+\mathbb{Z}_{\geq 0}\left(a_{2}, \text { wt } x_{2}\right)+\mathbb{Z}_{\geq 0}\left(a_{3}, \text { wt } x_{3}\right) \text { if } Y^{\sharp} \text { is smooth. }
\end{aligned}
$$

Note that $w_{Q}^{C}(0)=0$, and that $\left(r_{Q}, 0\right)$ is contained in the above semi-group since $C$ is smooth. Take a reflexive sheaf $\mathcal{L}$ on $Y$ which is isomorphic to the ideal sheaf defined by $x_{1}=0$ outside $Q$. We note that $\mathcal{L}^{\left[\otimes v_{Q}\right]} \cong \mathcal{O}_{Y}(E)$ and $\mathcal{L}^{\left[\otimes b_{Q}\right]} \cong \mathcal{O}_{Y}\left(K_{Y}\right)$ on the germ at $Q$, where $\mathcal{L}^{[\otimes i]}$ denotes the double dual of $\mathcal{L}^{\otimes i}$. Then for any integers 
$j_{1}, \ldots, j_{k}$ with $\sum_{1 \leq i \leq k} j_{i}=0$,

$$
\operatorname{Im}\left[\mathcal{L}^{\left[\otimes j_{1}\right]} \otimes \cdots \otimes \mathcal{L}^{\left[\otimes j_{k}\right]} \otimes \mathcal{O}_{C} \rightarrow \mathcal{O}_{C}\right]=\left(\mathfrak{m}_{Q \subset C}\right)^{\sum_{1 \leq i \leq k} w_{Q}^{C}\left(-j_{i}\right) / r_{Q}},
$$

where $\mathfrak{m}_{Q \subset C}$ is the ideal sheaf of $Q$ in $C$.

We also need to treat the sheaves $\mathcal{I}_{C} / \mathcal{I}_{C}^{(2)}$ as in $\left[17\right.$, Section 2]. For any $C \cong \mathbb{P}^{1} \subset$ $H \cap E$, its ideal sheaf as the subscheme in $Y$ is denoted by $\mathcal{I}_{C}$, and its symbolic 2-power by $\mathcal{I}_{C}^{(2)}$. Let $C^{(2)} \subset Y$ be the closed subscheme defined by $\mathcal{I}_{C}^{(2)}$. Then $C^{(2)} \subseteq 2 H \cap 2 E . H^{1}\left(\mathcal{O}_{2 H \cap 2 E}\right)=0$ unless $f$ is of type $\mathrm{O}$ or I, as in Lemma 2.8 whence $H^{1}\left(\mathcal{O}_{C^{(2)}}\right)=0$. By the exact sequence

$$
0 \rightarrow \mathcal{I}_{C} / \mathcal{I}_{C}^{(2)} \rightarrow \mathcal{O}_{C^{(2)}} \rightarrow \mathcal{O}_{C} \rightarrow 0,
$$

we obtain that $H^{1}\left(\mathcal{I}_{C} / \mathcal{I}_{C}^{(2)}\right)=0$ unless $f$ is of type $\mathrm{O}$ or I. On the other hand, consider the natural map

$$
\bigwedge^{2} \mathcal{I}_{C} / \mathcal{I}_{C}^{(2)} \otimes \mathcal{O}_{C}\left(K_{C}\right) \rightarrow\left[\mathcal{O}_{Y}\left(K_{Y}\right)\right]_{C}
$$

where $[\mathcal{L}]_{C}$ denotes the torsion-free part of $\mathcal{L} \otimes \mathcal{O}_{C}$. This map has cokernel of length $2+\operatorname{deg}\left[\mathcal{O}_{Y}\left(K_{Y}\right)\right]_{C}-\operatorname{deg} \bigwedge^{2} \mathcal{I}_{C} / \mathcal{I}_{C}^{(2)}$. Therefore we obtain the next result:

Lemma 2.10. The map (2.7) has cokernel of length $\leq 4+\operatorname{deg}\left[\mathcal{O}_{Y}\left(K_{Y}\right)\right]_{C}$ unless $f$ is of type $O$ or $I$.

The next lemma is obtained by following [17, Corollary 2.15] almost faithfully. Remark that $(2,2) \in \mathbb{Z} \times \mathbb{Z} /(4)$ is contained in the semi-group (2.5) when $Q$ is of exceptional type (iii) in Theorem 1.3 since $C$ is smooth at $Q$.

Lemma 2.11 ([17, Corollary 2.15]). Let $Q \in C \subset Y$ be a singular point of $Y$. Then the map (2.7) is not surjective at $Q$.

The following lemma shows how to compute of the length of the cokernel of the map (2.7) in the simplest case:

Lemma 2.12. Let $Q \in Y$ be a terminal quotient singularity $\mathbb{C}_{x_{1} x_{2} x_{3}}^{3} / \frac{1}{r}(1,-1, b)$, $Q \in C \subset Y$ a smooth curve, and $t$ a uniformising parameter with $\left.\left(x_{1}, x_{2}, x_{3}\right)\right|_{C}=$ $\left(t^{c / r}, t^{1-c / r}, 0\right)$ for some $0<c<r$. Then the map (2.7) has cokernel of length $\min \{c, r-c\}$.

Proof. First we give an explicit description of this map using local coordinates. The map $\mathcal{O}_{Y}\left(K_{Y}\right) \otimes \mathcal{O}_{C} \rightarrow\left[\mathcal{O}_{Y}\left(K_{Y}\right)\right]_{C} \cong \mathcal{O}_{C}$ can be locally given, but not naturally, by the map

$$
\begin{aligned}
& \mathcal{O}_{Y}\left(K_{Y}\right) \otimes \mathcal{O}_{C} \rightarrow \mathcal{O}_{C}, \\
& f\left(x_{1}, x_{2}, x_{3}\right) d x_{1} \wedge d x_{2} \wedge d x_{3} \mapsto \frac{f\left(t^{c / r}, t^{1-c / r}, 0\right)}{t^{w_{Q}^{C}(-b) / r}} .
\end{aligned}
$$

On the other hand $\mathcal{O}_{C}\left(K_{C}\right)$ is generated by $d\left(x_{1} x_{2}\right)$. Hence our map has cokernel of length equal to that of the composition of the map

$$
\begin{aligned}
\mathcal{I}_{C} / \mathcal{I}_{C}^{(2)} \times \mathcal{I}_{C} / \mathcal{I}_{C}^{(2)} & \rightarrow \mathcal{O}_{Y}\left(K_{Y}\right) \otimes \mathcal{O}_{C}, \\
f_{1} \times f_{2} & \mapsto d f_{1} \wedge d f_{2} \wedge d\left(x_{1} x_{2}\right)
\end{aligned}
$$

and the map (2.8).

It is easy to see $\mathcal{I}_{C} / \mathcal{I}_{C}^{(2)}$ is generated by functions of the form $g_{-b}\left(x_{1}, x_{2}\right) \cdot x_{3}$ and $g_{c}\left(x_{1}, x_{2}\right) \cdot\left(x_{1}^{r-c}-x_{2}^{c}\right)$ where $g_{-b}$ and $g_{c}$ are semi-invariant functions of weights 
$-b$, respectively $c$. Therefore we have only to consider the images of $g_{-b} d x_{3} \wedge$ $d\left(g_{c} \cdot\left(x_{1}^{r-c}-x_{2}^{c}\right)\right) \wedge d\left(x_{1} x_{2}\right)$ by the map (2.8). It suffices to consider those for $g_{c}=x_{1}^{c}$ and $x_{2}^{r-c}$. We have

$$
g_{-b} d x_{3} \wedge d\left(g_{c} \cdot\left(x_{1}^{r-c}-x_{2}^{c}\right)\right) \wedge d\left(x_{1} x_{2}\right)= \begin{cases}g_{-b} \cdot r x_{1}^{r} d x_{1} \wedge d x_{2} \wedge d x_{3} & \left(g_{c}=x_{1}^{c}\right), \\ g_{-b} \cdot r x_{2}^{r} d x_{1} \wedge d x_{2} \wedge d x_{3} & \left(g_{c}=x_{2}^{r-c}\right) .\end{cases}
$$

Since we can choose $g_{-b}$ so that $g_{-b}\left(t^{c / r}, t^{1-c / r}\right)=t^{w_{Q}^{C}(-b) / r}$, our length is the minimum of the orders of $x_{1}^{r}$ and $x_{2}^{r}$ with $\left(x_{1}, x_{2}\right)=\left(t^{c / r}, t^{1-c / r}\right)$ with respect to $t$, which is $\min \{c, r-c\}$.

We need to compute the length in the more complicated case below. Let $Q \in$ $Y$ be a terminal quotient singularity $\mathbb{C}_{x_{1} x_{2} x_{3}}^{3} / \frac{1}{r}(1,-1, b), Q \in C \subset Y$ a smooth curve, and $t$ a uniformising parameter with $\left.\left(x_{1}, x_{2}, x_{3}\right)\right|_{C}=\left(t^{a_{1} / r}, t^{a_{2} / r}, t^{a_{3} / r}\right)$. We can take an invariant monomial $g\left(x_{1}, x_{2}, x_{3}\right)$ so that $g\left(t^{a_{1} / r}, t^{a_{2} / r}, t^{a_{3} / r}\right)=t$. Then $\mathcal{I}_{C} / \mathcal{I}_{C}^{(2)}$ is generated by functions of the form $x_{1}^{s_{1}} x_{2}^{s_{2}} x_{3}^{s_{3}}-x_{1}^{t_{1}} x_{2}^{t_{2}} x_{3}^{t_{3}}$ with $\left(s_{1}, s_{2}, s_{3}\right) \neq\left(t_{1}, t_{2}, t_{3}\right)$ such that $s_{1}-s_{2}+b s_{3} \equiv t_{1}-t_{2}+b t_{3} \equiv 0$ modulo $r$ and $a_{1} s_{1}+a_{2} s_{2}+a_{3} s_{3}=a_{1} t_{1}+a_{2} t_{2}+a_{3} t_{3}$. Our length is the minimum of the orders of the images of $d\left(x_{1}^{s_{1}} x_{2}^{s_{2}} x_{3}^{s_{3}}-x_{1}^{t_{1}} x_{2}^{t_{2}} x_{3}^{t_{3}}\right) \wedge d\left(x_{1}^{\bar{s}_{1}} x_{2}^{\bar{s}_{2}} x_{3}^{\bar{s}_{3}}-x_{1}^{\bar{t}_{1}} x_{2}^{\bar{t}_{2}} x_{3}^{\bar{t}_{3}}\right) \wedge d g$ under the map

$$
\begin{aligned}
& \mathcal{O}_{Y}\left(K_{Y}\right) \otimes \mathcal{O}_{C} \rightarrow \mathcal{O}_{C}, \\
& f\left(x_{1}, x_{2}, x_{3}\right) d x_{1} \wedge d x_{2} \wedge d x_{3} \mapsto \frac{f\left(t^{a_{1} / r}, t^{a_{2} / r}, t^{a_{3} / r}\right)}{t^{w_{Q}^{C}(-b) / r}},
\end{aligned}
$$

where $x_{1}^{s_{1}} x_{2}^{s_{2}} x_{3}^{s_{3}}-x_{1}^{t_{1}} x_{2}^{t_{2}} x_{3}^{t_{3}}$ and $x_{1}^{\bar{s}_{1}} x_{2}^{\bar{s}_{2}} x_{3}^{\bar{s}_{3}}-x_{1}^{\bar{t}_{1}} x_{2}^{\bar{t}_{2}} x_{3}^{\bar{t}_{3}}$ satisfy the conditions mentioned above. The order for fixed $x_{1}^{s_{1}} x_{2}^{s_{2}} x_{3}^{s_{3}}-x_{1}^{t_{1}} x_{2}^{t_{2}} x_{3}^{t_{3}}$ and $x_{1}^{\bar{s}_{1}} x_{2}^{\bar{s}_{2}} x_{3}^{\bar{s}_{3}}-x_{1}^{\bar{t}_{1}} x_{2}^{\bar{t}_{2}} x_{3}^{\bar{t}_{3}}$ is $+\infty$ or

$$
\frac{1}{r} \cdot\left\{\left(a_{1} s_{1}+a_{2} s_{2}+a_{3} s_{3}\right)+\left(a_{1} \bar{s}_{1}+a_{2} \bar{s}_{2}+a_{3} \bar{s}_{3}\right)+r-\left(a_{1}+a_{2}+a_{3}+w_{Q}^{C}(-b)\right)\right\} .
$$

In particular, when we can take $g=x_{1} x_{2}$, it is $+\infty$ or

$$
\frac{1}{r} \cdot\left\{\left(a_{1} s_{1}+a_{2} s_{2}+a_{3} s_{3}\right)+\left(a_{1} \bar{s}_{1}+a_{2} \bar{s}_{2}+a_{3} \bar{s}_{3}\right)-\left(a_{3}+w_{Q}^{C}(-b)\right)\right\} .
$$

\section{LOCAL ANALYSIS AT A NON-GORENSTEIN POINT}

In this section we analyse the structure of $C \cong \mathbb{P}^{1} \subseteq H \cap E$ under the assumption that $f$ is of type II or III, focusing on the germ at a non-Gorenstein point. We can see the theorems in this section straightforwardly when $P$ is a smooth point because of the explicit description [7. Hence we here impose an extra assumption, that is, $P$ is a singular point. We have $(H \cdot C) \leq(H \cdot[H \cap E])=E^{3}$. By Theorem 1.4. Proposition 2.4(1) and Remarks 2.1 and 2.9, the configuration of the 1-cycle $[H \cap E]=\sum\left[C_{i}\right]$ is as follows:

Description 3.1. $\quad$ (i) (type IIa) $a=2$ or 4 and $Y$ has one non-Gorenstein point $Q$, through which any $C_{i}$ passes.

(a) $a=2$ and $H \cap E \cong \mathbb{P}^{1}$ scheme-theoretically.

(b) $a=2$ and $[H \cap E]=\left[\mathbb{P}^{1}\right]+\left[\mathbb{P}^{1}\right]$ is reducible or non-reduced.

(c) $a=4$ and $H \cap E \cong \mathbb{P}^{1}$ scheme-theoretically. 
(ii) (type $\left.\operatorname{IIb}^{\vee}\right) J=\{(r, 1),(r, 1)\}$ or $\{(2,1),(4,1)\}$ and $a=2$, respectively 3 . $Y$ has one non-Gorenstein point $Q . H \cap E \cong \mathbb{P}^{1}$ scheme-theoretically and passes through $Q$.

(iii) (type $\mathrm{IIb}^{\vee \vee}$ ) $a \leq\left(r_{1}+r_{2}\right) / 2$ and $Y$ has two non-Gorenstein points $Q_{1}$ and $Q_{2}$ of indices $r_{1}$ and $r_{2}$. Exactly one of the irreducible components of $(H \cap E)_{\text {red }}$ passes through both $Q_{1}$ and $Q_{2}$, and all the others pass through only $Q_{2}$.

(iv) (type III) $a \leq(1+r) / 2$ and $Y$ has one non-Gorenstein point $Q$, through which any $C_{i}$ passes.

Let $s_{C}(i)$ be the integer such that $\left[\mathcal{O}_{Y}(i E)\right]_{C} \cong \mathcal{O}_{\mathbb{P}^{1}}\left(s_{C}(i)\right)$. The following is easy to see by Lemmata 2.8 and 3.3 and Remark 2.1 ;

Lemma 3.2. $\quad$ (i) Assume that $C$ comes from type IIa. Then

(a) $s_{C}(1)=\cdots=s_{C}(a-1)=-1$.

(b) $s_{C}(-i) \geq-1$ for $i \geq 1$.

(c) $s_{C}(a)=-2$ in Description 3.1(ia), (ic), and $s_{C}(a)=-2$ or -1 in Description 3.1(ib).

(ii) Assume that $C$ comes from type $I I b^{\vee}$. Then

(a) $s_{C}(1)=\cdots=s_{C}(a-1)=-1 . s_{C}(a)=-2$.

(b) $s_{C}(-i) \geq-1$ for $i \geq 1$.

(iii) Assume that $C$ comes from type $I I b^{\vee \vee}$ with $Q_{1}, Q_{2} \in C$. Then

(a) $s_{C}(1)=\cdots=s_{C}(a-1)=-1$.

(b) $s_{C}(-i)=-1$ for $1 \leq i \leq a-1, r_{1} \nmid i$.

(c) $s_{C}\left(-r_{1} i\right)=0$ or -1 for $1 \leq r_{1} i \leq a-1$.

(d) $\left(s_{C}(a), s_{C}(-a)\right)=(-2,0),(-2,-1)$ or $(-1,-1)$.

(iv) Assume that $C$ comes from type III. Then

(a) $s_{C}(1)=\cdots=s_{C}(a-1)=-1$.

(b) $s_{C}(-1)=\cdots=s_{C}(-(a-1))=0$.

(c) $\left(s_{C}(a), s_{C}(-a)\right)=(-2,1),(-2,0)$ or $(-1,0)$.

Proof. For instance, we consider the case (iv). $s_{C}(i)<0$ for $i \geq 1$ by the existence of the natural map $\mathcal{O}_{Y}(i E)^{\otimes r} \otimes \mathcal{O}_{C} \rightarrow \mathcal{O}_{Y}(i r E) \otimes \mathcal{O}_{C}$, so $s_{C}(1)=\cdots=s_{C}(a-1)=$ $-1, s_{C}(a)=-2$ or -1 by Lemma 2.8 . On the other hand, Lemma 3.3 with $\mathcal{L}=\mathcal{O}_{Y}(E)$ implies that $s_{C}(-1)=0$, so $s_{C}(-i) \geq 0$ for $i \geq 1$ by the existence of the natural map $\mathcal{O}_{Y}(-E)^{\otimes i} \otimes \mathcal{O}_{C} \rightarrow \mathcal{O}_{Y}(-i E) \otimes \mathcal{O}_{C}$. Hence $s_{C}(-1)=\cdots=$ $s_{C}(-(a-1))=0$ and $\left(s_{C}(a), s_{C}(-a)\right)=(-2,1),(-2,0)$ or $(-1,0)$, because the map $\mathcal{O}_{Y}(-i E) \otimes \mathcal{O}_{Y}(i E) \otimes \mathcal{O}_{C} \rightarrow \mathcal{O}_{C}$ is not surjective for $1 \leq i<r$ and $a<r$ by Remark 2.1

Lemma 3.3. Let $Q \in Y$ be a terminal quotient singularity $\mathbb{C}_{x_{1} x_{2} x_{3}}^{3} / \frac{1}{r}(1,-1, b)$, and $\mathcal{L}$ a reflexive sheaf on $Y$ which is isomorphic to the ideal sheaf defined by $x_{1}=0$ outside $Q$. Then the image of the natural map $\mathcal{L} \otimes \mathcal{L}^{[-1]} \rightarrow \mathcal{O}_{Y}$ is the ideal sheaf of $Q$ in $Y$.

Proof. It is enough to show that any invariant monomial $\neq 1$ of $x_{1}, x_{2}, x_{3}$ decomposes into two semi-invariant monomials with weights 1 and -1 , but it is trivial since $b$ is co-prime to $r$.

The following lemma stores more information on $s_{C}$ : 
Lemma 3.4. Assume that $f$ is not of type $O$ or $I$, and take a curve $C \cong \mathbb{P}^{1} \subseteq H \cap E$. Let $i$ be a positive integer, and $T, T^{\prime}$ surfaces on $Y$ defined by general elements in $\mathcal{O}_{Y}(-i E)$.

(i) Assume that $\chi\left(\mathcal{Q}_{-i}\right) \geq 2$. Then $(T \cap E)_{\text {red }}$ has an irreducible component which properly intersects $\left(T^{\prime} \cap E\right)_{\mathrm{red}}$.

(ii) Assume that $\chi\left(\mathcal{Q}_{-j}\right)=1$ for $1 \leq j<i$ and $\chi\left(\mathcal{Q}_{-i}\right) \geq 2$. If $C=H \cap E$ scheme-theoretically, then $(T \cap E)_{\mathrm{red}}$ properly intersects $\left(T^{\prime} \cap E\right)_{\mathrm{red}}$ and C. Moreover, $s_{C}(-j)=-1$ for $1 \leq j<i$ and $s_{C}(-i) \geq 0$.

Proof. (1i) By (2.3), we have a surjective map $f_{*} \mathcal{O}_{Y}(-i E) \rightarrow H^{0}\left(\mathcal{Q}_{-i}\right)$. Write $E^{o}$ for the restriction of the Gorenstein locus of $Y$ to $E$. Take a resolution $\hat{E}$ of $E$ and let $\hat{E}^{o}$ be the pre-image of $E^{o}$. Note that $H^{0}\left(\left.\mathcal{Q}_{-i}\right|_{E^{o}}\right)=H^{0}\left(\mathcal{Q}_{-i}\right)$ by (2.1). Consider a linear system $L$ on $\hat{E}$, not necessarily complete, whose restriction to $\hat{E}^{o}$ is $H^{0}\left(\left.\mathcal{Q}_{-i}\right|_{E^{o}}\right) . h^{0}\left(\mathcal{Q}_{-i}\right)=\chi\left(\mathcal{Q}_{-i}\right) \geq 2$ means that $L$ moves, and its general member has an irreducible component which does not appear in the union of the support of the fixed locus of $L$ and the pre-image of the non-normal locus of $E$. The element in $H^{0}\left(\mathcal{Q}_{-i}\right)$ corresponding to this member gives the desired section $T$.

(iii) By the commutative diagrams in Section 2 and Lemma 2.8(1i), we have surjective maps

$$
f_{*} \mathcal{O}_{Y}(-j E) \rightarrow H^{0}\left(\mathcal{Q}_{-j}\right) \rightarrow H^{0}\left(\mathcal{R}_{-j}\right) \rightarrow H^{0}\left(\left[\mathcal{O}_{Y}(-j E)\right]_{C}\right) \quad(1 \leq j \leq i) .
$$

Remark that $E$ is smooth at the generic point of $C$ because $C=H \cap E$ schemetheoretically. $h^{0}\left(\mathcal{Q}_{-j}\right)=\chi\left(\mathcal{Q}_{-j}\right)=1$ for $1 \leq j<i$ and a non-zero element in $H^{0}\left(\mathcal{Q}_{-j}\right)$ corresponds to, at the generic point of $C$, the Cartier divisor defined by $j H$. Hence the map $H^{0}\left(\mathcal{Q}_{-j}\right) \rightarrow H^{0}\left(\left[\mathcal{O}_{Y}(-j E)\right]_{C}\right)$ is a zero map and we obtain $s_{C}(-j)=-1$ by Lemma[2.8(iii). On the other hand, the linear system $L$ constructed from $H^{0}\left(\mathcal{Q}_{-i}\right)$ as in the proof of (ii) moves. Because $h^{0}\left(\mathcal{Q}_{-j}\right)=1$ for $1 \leq j<i$ and $E$ is smooth at the generic point of $C$, its general member does not have the birational transform of $C$ as its component. Thus we have the desired section and the map $H^{0}\left(\mathcal{Q}_{-i}\right) \rightarrow H^{0}\left(\left[\mathcal{O}_{Y}(-i E)\right]_{C}\right)$ is a non-zero map, whence $s_{C}(-i) \geq 0$.

First we treat type IIa.

Theorem 3.5. Assume that $f$ is of type IIa. Let $Q \in C \cong \mathbb{P}^{1} \subseteq H \cap E$ be the non-Gorenstein point with an irreducible component of $H \cap E$ as in Description 3.1(1i). Take a normal form of $Q \in C \subset Y$ as in Section [2]

(i) The case (ia) in Description 3.1 does not happen.

(ii) In the case (ib) in Description 3.1, we have $s_{C}(-2)=0$ and $\left(a_{1}, a_{2}, a_{3}\right)=$ $((r+1) / 2,(r-1) / 2,2)$. In particular, we may choose semi-invariant local coordinates $x_{1}, x_{2}, x_{3}$ with weights $\mathrm{wt}\left(x_{1}, x_{2}, x_{3}\right)=(1,-1,4)$ of the indexone cover $Q^{\sharp} \in Y^{\sharp}$ so that $\left.\left(x_{1}, x_{2}, x_{3}\right)\right|_{C^{\dagger}}=\left(t^{(r+1) / 2 r}, 0, t^{2 / r}\right)$ when $r \equiv 1$ modulo 4 , and $\left(0, t^{(r-1) / 2 r}, t^{2 / r}\right)$ when $r \equiv 3$ modulo 4 .

(iii) In the case (ic) in Description 3.1, we have $r=5, s_{C}(-4)=0$ and $\left(a_{1}, a_{2}, a_{3}\right)=(3,2,4)$. In particular, we may choose semi-invariant local coordinates $x_{1}, x_{2}, x_{3}$ with weights $\mathrm{wt}\left(x_{1}, x_{2}, x_{3}\right)=(1,-1,3)$ of the indexone cover $Q^{\sharp} \in Y^{\sharp}$ so that $\left.\left(x_{1}, x_{2}, x_{3}\right)\right|_{C^{\dagger}}=\left(t^{3 / 5}, t^{2 / 5}, 0\right)$.

Proof. (ii). Compute the image of the map

$$
\mathcal{O}_{Y}(E)^{\otimes r} \otimes \mathcal{O}_{Y}(-r E) \otimes \mathcal{O}_{C} \rightarrow \mathcal{O}_{C}
$$


by (2.6). It is $\left(\mathfrak{m}_{Q \subset C}\right)^{w_{Q}^{C}(-2)}$. On the other hand, this image equals that of

$$
\left[\mathcal{O}_{Y}(E)\right]_{C}^{\otimes r} \otimes\left[\mathcal{O}_{Y}(-r E)\right]_{C} \rightarrow \mathcal{O}_{C}
$$

whence $r s_{C}(1)-r(E \cdot C)=-w_{Q}^{C}(-2)$. By $s_{C}(1)=-1$ in Lemma 3.2(1) and $(H \cdot C)=E^{3}=2 / r$ in Theorem 1.4 we have $w_{Q}^{C}(-2)=r-2$. Hence we can write $\left(a_{1}, a_{2}, a_{3}\right)=\left(1+r m_{1}, r-1+r m_{2}, 4+r m_{3}\right)$ with $m_{1}, m_{2}, m_{3} \in \mathbb{Z}_{\geq 0}$ by (2.4). We see that $m_{1}=0$ easily since there exists an invariant monomial of $x_{1}, x_{2}, x_{3}$ whose restriction to $C$ is $t$. Thus $\left(a_{1}, a_{2}, a_{3}\right)=(1, r-1,4)$ and $w_{Q}^{C}(-4)=$ $r-4$. Consider another map $\mathcal{O}_{Y}(2 E)^{\otimes r} \otimes \mathcal{O}_{Y}(-2 r E) \otimes \mathcal{O}_{C} \rightarrow \mathcal{O}_{C}$. Then we have $r s_{C}(2)-2 r(E \cdot C)=-w_{Q}^{C}(-4)$, whence $s_{C}(2)=-1$ by $w_{Q}^{C}(-4)=r-4$ and $(H \cdot C)=2 / r$. But this contradicts Lemma 3.2 (i) .

(ii) By the map $\mathcal{O}_{Y}(E)^{\otimes r} \otimes \mathcal{O}_{Y}(-r E) \otimes \mathcal{O}_{C} \rightarrow \mathcal{O}_{C}$, we have $r s_{C}(1)-r(E \cdot C)=$ $-w_{Q}^{C}(-2)$. Hence $w_{Q}^{C}(-2)=r-1$ by Lemma 3.2(1) and $(H \cdot C)=E^{3} / 2=1 / r$ in Theorem 1.4. and we can write $\left(a_{1}, a_{2}, a_{3}\right)=\left((r+1) / 2+r m_{1},(r-1) / 2+r m_{2}\right.$, $\left.2+r m_{3}\right)$ with $m_{1}, m_{2}, m_{3} \in \mathbb{Z}_{\geq 0}$ by (2.4). Because there exists an invariant monomial of $x_{1}, x_{2}, x_{3}$ whose restriction to $C$ is $t$, one of the following holds:

(i) $(r, 0) \in \mathbb{Z}_{>0}\left(a_{1}, 1\right)+\mathbb{Z}_{>0}\left(a_{3}, 4\right)$ or $\in \mathbb{Z}_{>0}\left(a_{2},-1\right)+\mathbb{Z}_{>0}\left(a_{3}, 4\right)$ in $\mathbb{Z} \times \mathbb{Z} /(r)$. (ii) $r=a_{1}+a_{2}$.

If (ii) holds, $\left(a_{1}, a_{2}, a_{3}\right)=((r+1) / 2,(r-1) / 2,2)$ and $w_{Q}^{C}(4)=4$. By the map $\mathcal{O}_{Y}(-2 E)^{\otimes r} \otimes \mathcal{O}_{Y}(2 r E) \otimes \mathcal{O}_{C} \rightarrow \mathcal{O}_{C}$, we have $r s_{C}(-2)+2 r(E \cdot C)=-w_{Q}^{C}(4)$, whence $s_{C}(-2)=0$. Now we assume (iii). Then $\left(a_{1}, a_{2}, a_{3}\right)=((r+1) / 2$, $\left.(r-1) / 2,2+r m_{3}\right)$ with some $m_{3}$. We have nothing to do if $m_{3}=0$, so we also assume that $m_{3}>0$ and $r \geq 7$. Note that $m_{3} \leq 2$ by $4 a_{1}=2+2 r$.

Suppose that $m_{3}=2$. We may choose semi-invariant local coordinates $x_{1}, x_{2}, x_{3}$ with weights $\operatorname{wt}\left(x_{1}, x_{2}, x_{3}\right)=(1,-1,4)$ of $Q^{\sharp} \in Y^{\sharp}$ so that $\left.\left(x_{1}, x_{2}, x_{3}\right)\right|_{C^{\dagger}}=$ $\left(t^{(r+1) / 2 r}, t^{(r-1) / 2 r}, 0\right)$. We see that $w_{Q}^{C}(-4)=4 w_{Q}^{C}(-1)=2 r-2$ by $r \geq 7$. Considering the map $\mathcal{O}_{Y}(2 E)^{\otimes r} \otimes \mathcal{O}_{Y}(-2 r E) \otimes \mathcal{O}_{C} \rightarrow \mathcal{O}_{C}$, we obtain $r s_{C}(2)-2 r(E \cdot C)=$ $-w_{Q}^{C}(-4)$, whence $s_{C}(2)=-2$. By Lemma 2.10, the map (2.7) has cokernel of length $\leq 2$ at $Q$. Thus $(r-1) / 2 \leq 2$ by Lemma 2.12 which contradicts $r \geq 7$.

It remains to exclude the case where $\left(a_{1}, a_{2}, a_{3}\right)=((r+1) / 2,(r-1) / 2$, $r+2)$ with $r \geq 7$. But if $r=7$, then $\left(a_{1}, a_{2}, a_{3}\right)=(4,3,9)$ and we may choose the coordinates so that $\left.\left(x_{1}, x_{2}, x_{3}\right)\right|_{C^{\dagger}}=\left(t^{4 / 7}, t^{3 / 7}, 0\right)$. Then $s_{C}(2)=-2$ and it contradicts Lemmata 2.10 and 2.12 Therefore we may assume that $r \geq 9$. By the $\operatorname{map} \mathcal{O}_{Y}(-3 E)^{\otimes r} \otimes \mathcal{O}_{Y}(3 r E) \otimes \mathcal{O}_{C} \rightarrow \mathcal{O}_{C}$, we have $r s_{C}(-3)+3 r(E \cdot C)=-w_{Q}^{C}(6)$, whence $w_{Q}^{C}(6)=3$ or $r+3$ by $s_{C}(-3) \geq-1$. From $\left(a_{1}, a_{2}, a_{3}\right)=((r+1) / 2$, $(r-1) / 2, r+2)$ with $r \geq 9, r$ has to be 9 . When $r=9,\left(a_{1}, a_{2}, a_{3}\right)=(5,4,11)$ and $w_{Q}^{C}(-4)=16$. By the map $\mathcal{O}_{Y}(2 E)^{\otimes r} \otimes \mathcal{O}_{Y}(-18 E) \otimes \mathcal{O}_{C} \rightarrow \mathcal{O}_{C}$, we have $9 s_{C}(2)-$ $18(E \cdot C)=-w_{Q}^{C}(-4)$, whence $s_{C}(2)=-2$. We consider $x_{1}^{s_{1}} x_{2}^{s_{2}} x_{3}^{s_{3}}-x_{1}^{t_{1}} x_{2}^{t_{2}} x_{3}^{t_{3}}$ with $\left(s_{1}, s_{2}, s_{3}\right) \neq\left(t_{1}, t_{2}, t_{3}\right)$ such that $s_{1}-s_{2}+4 s_{3} \equiv t_{1}-t_{2}+4 t_{3} \equiv 0$ modulo 9 and $5 s_{1}+4 s_{2}+11 s_{3}=5 t_{1}+4 t_{2}+11 t_{3}$. We can easily see that $\left(5 s_{1}+4 s_{2}+11 s_{3}\right) / 9 \geq 3$ for any such $x_{1}^{s_{1}} x_{2}^{s_{2}} x_{3}^{s_{3}}-x_{1}^{t_{1}} x_{2}^{t_{2}} x_{3}^{t_{3}}$. Hence by the last paragraph in Section 2, the map (2.7) has cokernel of length $\geq 3+3-\left(a_{3}+w_{Q}^{C}(-4)\right) / 9=3$. It contradicts Lemma 2.10] and $s_{C}(2)=-2$.

(iii) Consider the maps $\mathcal{O}_{Y}(i E)^{\otimes r} \otimes \mathcal{O}_{Y}(-i r E) \otimes \mathcal{O}_{C} \rightarrow \mathcal{O}_{C}$ for $i=1,2,3,4$. Then we have $r s_{C}(i)-i r(E \cdot C)=-w_{Q}^{C}(-2 i)$, whence $w_{Q}^{C}(-2 i)=r-i$ for $i=1,2,3$ and $w_{Q}^{C}(-8)=2 r-4$ by Lemma 3.2 (ic). We can write $\left(a_{1}, a_{2}, a_{3}\right)=((r+1) / 2+$ $\left.r m_{1},(r-1) / 2+r m_{2}, 4+r m_{3}\right)$ with $m_{1}, m_{2}, m_{3} \in \mathbb{Z}_{\geq 0}$ by (2.4). If $r=5$, then 
$a_{1}=w_{Q}^{C}(-4)=3$ and $a_{2}=w_{Q}^{C}(-6)=2$ and we have the desired description. In this case, we have $w_{Q}^{C}(8)=4$. By the map $\mathcal{O}_{Y}(-4 E)^{\otimes r} \otimes \mathcal{O}_{Y}(4 r E) \otimes \mathcal{O}_{C} \rightarrow \mathcal{O}_{C}$, we obtain $r s_{C}(-4)+4 r(E \cdot C)=-w_{Q}^{C}(8)$, whence $s_{C}(-4)=0$.

We derive a contradiction supposing that $r \geq 7$. Since $w_{Q}^{C}(-4)=r-2$, we can decompose $r-2$ into $(r+1) / 2+4 c$ or $(r-1) / 2+4 c$ for some $c>0$. Then $r \equiv 5$, respectively $3(\bmod 8)$. Since $w_{Q}^{C}(-6)=r-3$, similarly we can decompose $r-3$ into $(r+1) / 2+4 c,(r-1) / 2+4 c$ or $4 c$. Then $r \equiv 7(\bmod 8), r \equiv 5(\bmod 8)$ or $r \equiv 3$ $(\bmod 4)$ respectively. Hence $r \equiv 3$ or $5(\bmod 8)$ with $m_{2}=m_{3}=0$, and $r \geq 11$. $\left(a_{1}, a_{2}, a_{3}\right)=((r+1) / 2,(r-1) / 2,4)$, since $(r, 0)$ is contained in the semi-group (2.5). We consider $x_{1}^{s_{1}} x_{2}^{s_{2}} x_{3}^{s_{3}}-x_{1}^{t_{1}} x_{2}^{t_{2}} x_{3}^{t_{3}}$ with $\left(s_{1}, s_{2}, s_{3}\right) \neq\left(t_{1}, t_{2}, t_{3}\right)$ such that $s_{1}-s_{2}+8 s_{3} \equiv t_{1}-t_{2}+8 t_{3} \equiv 0$ modulo $r$ and $a_{1} s_{1}+a_{2} s_{2}+a_{3} s_{3}=a_{1} t_{1}+a_{2} t_{2}+a_{3} t_{3}$. Then $\left(a_{1} s_{1}+a_{2} s_{2}+a_{3} s_{3}\right) / r=2$ only if each of $x_{1}^{s_{1}} x_{2}^{s_{2}} x_{3}^{s_{3}}$ and $x_{1}^{t_{1}} x_{2}^{t_{2}} x_{3}^{t_{3}}$ is $x_{1}^{2} x_{2}^{2}$, $x_{1} x_{3}^{(3 r-1) / 8}$ with $r \equiv 3(\bmod 8)$, or $x_{2} x_{3}^{(3 r+1) / 8}$ with $r \equiv 5(\bmod 8)$, and otherwise $\left(a_{1} s_{1}+a_{2} s_{2}+a_{3} s_{3}\right) / r \geq 3$. Hence by the last paragraph in Section 2, the map (2.7) has cokernel of length $\geq 2+3-\left(a_{3}+w_{Q}^{C}(-8)\right) / r=3$. It contradicts Lemma 2.10 and $s_{C}(4)=-2$ in Lemma 3.2(1).

It is easy to see the local structure in type IIb ${ }^{\vee}$.

Theorem 3.6. Assume that $f$ is of type $I I b^{\vee}$. Let $Q \in C=H \cap E \cong \mathbb{P}^{1}$ be the non-Gorenstein point as in Description 3.11ii). Take a normal form of $Q \in C \subset Y$ as in Section 2 .

(i) If $J=\{(r, 1),(r, 1)\}$, then $a=2, s_{C}(-2)=0$ and $\left(a_{1}, a_{2}, a_{3}, a_{4}\right)=(r+$ $1, r-1,2, r)$. In particular, we may choose semi-invariant local coordinates $x_{1}, x_{2}, x_{3}, x_{4}$ with weights $\operatorname{wt}\left(x_{1}, x_{2}, x_{3}, x_{4}\right)=(1,-1,2,0)$ of the index-one cover $Q^{\sharp} \in Y^{\sharp}$ so that $\left.\left(x_{1}, x_{2}, x_{3}, x_{4}\right)\right|_{C^{\dagger}}=\left(0,0, t^{2 / r}, t\right)$.

(ii) If $J=\{(2,1),(4,1)\}$, then $a=3, s_{C}(-3)=0$ and $\left(a_{1}, a_{2}, a_{3}, a_{4}\right)=$ $(5,3,3,2)$. In particular, we may choose semi-invariant local coordinates $x_{1}, x_{2}, x_{3}, x_{4}$ with weights $\operatorname{wt}\left(x_{1}, x_{2}, x_{3}, x_{4}\right)=(1,3,3,2)$ of the index-one cover $Q^{\sharp} \in Y^{\sharp}$ so that $\left.\left(x_{1}, x_{2}, x_{3}, x_{4}\right)\right|_{C^{\dagger}}=\left(0,0, t^{3 / 4}, t^{1 / 2}\right)$.

Proof. (1i) First applying Lemma 3.4(ii) with $i=2$ by Remark 2.5(1ii), we have $s_{C}(-1)=-1$ and $s_{C}(-2) \geq 0$. By the map $\mathcal{O}_{Y}(-2 E) \otimes \mathcal{O}_{Y}(E) \otimes \mathcal{O}_{C} \rightarrow \mathcal{O}_{Y}(-E) \otimes$ $\mathcal{O}_{C}$, we have $s_{C}(-2)+s_{C}(1) \leq s_{C}(-1)$, whence $s_{C}(-2)=0$. By the map $\mathcal{O}_{Y}(-E)^{\otimes r} \otimes \mathcal{O}_{Y}(r E) \otimes \mathcal{O}_{C} \rightarrow \mathcal{O}_{C}$, we have $r s_{C}(-1)+r(E \cdot C)=-w_{Q}^{C}(1)$. By $s_{C}(-1)=-1$ and $E^{3}=1 / r$, we obtain $w_{Q}^{C}(1)=1+r$. Thus we can write $\left(a_{1}, a_{2}, a_{3}, a_{4}\right)=\left(r+1+r m_{1}, r-1+r m_{2}, 2+r m_{3}, r\right)$ with $m_{1}, m_{2}, m_{3} \in \mathbb{Z}_{\geq 0}$ by (2.4) and the fact that $(r, 0)$ is contained in the semi-group (2.5). Consider another map $\mathcal{O}_{Y}(-2 E)^{\otimes r} \otimes \mathcal{O}_{Y}(2 r E) \otimes \mathcal{O}_{C} \rightarrow \mathcal{O}_{C}$. Then we have $r s_{C}(-2)+2 r(E \cdot C)=-w_{Q}^{C}(2)$. Therefore $w_{Q}^{C}(2)=2$ from $s_{C}(-2)=0$, whence $\left(a_{1}, a_{2}, a_{3}, a_{4}\right)=(r+1, r-1,2, r)$.

(iii) Applying Lemma 3.4(iii) with large $i$ by Remark 2.5(iii), we obtain $s_{C}(-1)=$ -1. By the map $\mathcal{O}_{Y}(-E)^{\otimes 4} \otimes \mathcal{O}_{Y}(4 E) \otimes \mathcal{O}_{C} \rightarrow \mathcal{O}_{C}$, we have $4 s_{C}(-1)+4(E \cdot C)=$ $-w_{Q}^{C}(1)$. By $s_{C}(-1)=-1$ and $E^{3}=1 / 4$, we have $w_{Q}^{C}(1)=5$. Hence we can write $\left(a_{1}, a_{2}, a_{3}, a_{4}\right)=\left(5+4 m_{1}, 3+4 m_{2}, 3+4 m_{3}, 2\right)$ with $m_{1}, m_{2}, m_{3} \in \mathbb{Z}_{>0}$. Consider another map $\mathcal{O}_{Y}(E)^{\otimes 4} \otimes \mathcal{O}_{Y}(-4 E) \otimes \mathcal{O}_{C} \rightarrow \mathcal{O}_{C}$. Then we have $4 s_{C}(1)-$ $4(E \cdot C)=-w_{Q}^{C}(-1)$. Thus $w_{Q}^{C}(-1)=3$ by Lemma 3.2(ii), whence $\left(a_{1}, a_{2}, a_{3}, a_{4}\right)=$ $(5,3,3,2)$. Furthermore, $w_{Q}^{C}(3)=3$. By the map $\mathcal{O}_{Y}(-3 E)^{\otimes 4} \otimes \mathcal{O}_{Y}(12 E) \otimes \mathcal{O}_{C} \rightarrow$ $\mathcal{O}_{C}$, we have $4 s_{C}(-3)+12(E \cdot C)=-w_{Q}^{C}(3)$, whence $s_{C}(-3)=0$. 
It is hardest to treat types $\operatorname{IIb}^{\vee \vee}$ and III.

Description 3.7. (i) If $f$ is of type $\mathrm{IIb}^{\vee \vee}$, there exists the unique irreducible component $C \cong \mathbb{P}^{1} \subseteq H \cap E$ through $Q_{1}$ and $Q_{2}$. We do not consider any other components from now on. Take normal forms of $Q_{i} \in C \subset Y$ for $i=1,2$ as in Section 2, using local coordinates $x_{i 1}, x_{i 2}, x_{i 3}$ and numbers $a_{i 1}, a_{i 2}, a_{i 3}$ respectively.

(ii) If $f$ is of type III, we take any irreducible component $C \cong \mathbb{P}^{1} \subseteq H \cap E$ and a normal form of $Q \in C \subset Y$ as in Section 2

Lemma 3.8. Assume that $s_{C}(-a)=0$ in Description 3.7(1), or $s_{C}(-a)=1$ in Description 3.7(1ii). Then $C=H \cap E$ scheme-theoretically and $s_{C}(a)=-2$. Moreover, for any non-Gorenstein point $Q \in Y$ with $Q \in C \cong \mathbb{P}^{1}=H \cap E$ in Description 3.7 we may choose semi-invariant local coordinates $x_{1}, x_{2}, x_{3}$ with weights $\operatorname{wt}\left(x_{1}, x_{2}, x_{3}\right)=(1,-1, a)$ of the index-one cover $Q^{\sharp} \in Y^{\sharp}$ so that

$$
Q^{\sharp} \in C^{\sharp} \subset Y^{\sharp} \cong o \in\left(x_{3} \text {-axis }\right) \subset \mathbb{C}_{x_{1} x_{2} x_{3}}^{3} .
$$

Proof. By the map $\mathcal{O}_{Y}(-a E)^{\otimes r} \otimes \mathcal{O}_{Y}(a r E) \otimes \mathcal{O}_{C} \rightarrow \mathcal{O}_{C}$, we have

$$
r s_{C}(-a)+\operatorname{ar}(E \cdot C)= \begin{cases}-r w_{Q_{1}}^{C}(a) / r_{1}-r w_{Q_{2}}^{C}(a) / r_{2} & \text { (Description 3.7(1i)), } \\ -w_{Q}^{C}(a) & \text { (Description 3.7(1ii)). }\end{cases}
$$

Therefore by Theorem 1.4,

$$
\begin{aligned}
\frac{w_{Q_{1}}^{C}(a)}{r_{1}}+\frac{w_{Q_{2}}^{C}(a)}{r_{2}} & =a(H \cdot C) \leq \frac{1}{r_{1}}+\frac{1}{r_{2}} & & \text { (Description 3.7(ii)) } \\
\frac{w_{Q}^{C}(a)}{r} & =a(H \cdot C)-1 \leq \frac{1}{r} & & \text { (Description 3.7(iii)). }
\end{aligned}
$$

Hence $C=H \cap E$ by Remark 2.9, and $w_{Q}^{C}(a)=1$ for any $Q \in C$ in Description 3.7. Therefore we have the desired description. $s_{C}(a)=-2$ follows from Lemma 3.2 .

Lemma 3.9. Assume that $f$ is of type $I I b^{\vee \vee}$ or III. Consider any non-Gorenstein point $Q \in Y$ with $Q \in C \cong \mathbb{P}^{1} \subseteq H \cap E$ given in Description [3.7. Then one of the following holds:

(i) One of $\left(a_{1}, 1\right)$ and $\left(a_{2},-1\right)$ is generated by the other and $\left(a_{3}, a\right)$ in $\mathbb{Z} \times$ $\mathbb{Z} /\left(r_{Q}\right)$. We may choose semi-invariant local coordinates $x_{1}, x_{2}, x_{3}$ with weight $\operatorname{wt}\left(x_{1}, x_{2}, x_{3}\right)=(1,-1, a)$ of the index-one cover $Q^{\sharp} \in Y^{\sharp}$ so that $\left.\left(x_{1}, x_{2}, x_{3}\right)\right|_{C^{\dagger}}=\left(0, t^{c_{Q} / r_{Q}}, t^{1-\overline{a c_{Q}} / r_{Q}}\right)$ or $\left(t^{c_{Q} / r_{Q}}, 0, t^{\overline{a_{Q}} / r_{Q}}\right)$ for some $0<$ $c_{Q}<r_{Q}$.

(ii) $f$ is of type III, $a=2, r \geq 5, s_{Q}(2)=-2$, and $\left(a_{1}, a_{2}, a_{3}\right)=(2, r-2,4)$ or $(r-2,2,2 r-4)$. In particular, we may choose semi-invariant local coordinates $x_{1}, x_{2}, x_{3}$ with weights wt $\left(x_{1}, x_{2}, x_{3}\right)=(1,-1,2)$ of the index-one cover $Q^{\sharp} \in Y^{\sharp}$ so that $\left.\left(x_{1}, x_{2}, x_{3}\right)\right|_{C^{\dagger}}=\left(t^{2 / r}, t^{1-2 / r}, 0\right)$ or $\left(t^{1-2 / r}, t^{2 / r}, 0\right)$. $(H \cdot C)$ equals $2 / r$, respectively $1-2 / r$.

Proof. Recall that there exists an invariant monomial in $x_{1}, x_{2}, x_{3}$ whose restriction to $C$ is $t$. Thus one of the following holds:

(i) One of $a_{1}, a_{2}, a_{3}$ is 1 .

(ii) $\left(r_{Q}, 0\right) \in \mathbb{Z}_{>0}\left(a_{1}, 1\right)+\mathbb{Z}_{>0}\left(a_{3}, a\right)$, or $\in \mathbb{Z}_{>0}\left(a_{2},-1\right)+\mathbb{Z}_{>0}\left(a_{3}, a\right)$ in $\mathbb{Z} \times$ $\mathbb{Z} /\left(r_{Q}\right)$. 
(iii) $r_{Q}=a_{1}+a_{2}$.

If (ii) or (ii) happens, one of $\left(a_{1}, 1\right)$ and $\left(a_{2},-1\right)$ is generated by the other and $\left(a_{3}, a\right)$. Thus, from now on we may assume that only (iii) of the above holds. By permutation of $x_{i}$, we may also assume that $r_{Q} \geq 5$. Then $w_{Q}^{C}(i)+w_{Q}^{C}(-i) \neq r_{Q}$ for $i=2,3$, whence the image of the map $\left[\mathcal{O}_{Y}(-i E)\right]_{C} \otimes\left[\mathcal{O}_{Y}(i E)\right]_{C} \rightarrow \mathcal{O}_{C}$ at $Q$ is properly contained in $\mathfrak{m}_{Q \subset C}$ for $i=2,3$ by 2.6. By Lemma 3.2 one of the following occurs:

(i) $a=2, s_{C}(2)=-2$, and $s_{C}(-2)=-1$ in Description 3.7 (i) or $s_{C}(-2)=0$ in Description 3.7 (ii).

(ii) $Q=Q_{2}, a=3, r_{1}=2, s_{C}(3)=-2$, and $s_{C}(-2)=s_{C}(-3)=-1$ in Description 3.7(ii).

Consider the case (1i). $w_{Q}^{C}(1)+w_{Q}^{C}(-1)=r_{Q}$ and $w_{Q}^{C}(2)+w_{Q}^{C}(-2)=2 r_{Q}$, whence $w_{Q}^{C}(2)=2 w_{Q}^{C}(1)$ and $w_{Q}^{C}(-2)=2 w_{Q}^{C}(-1)$. We have $\left(a_{1}, a_{2}, a_{3}\right)=\left(c, r_{Q}-c, 2 c\right)$ by setting $w_{Q}^{C}(1)=c$. Thus we may choose semi-invariant local coordinates $x_{1}, x_{2}, x_{3}$ with weights $\operatorname{wt}\left(x_{1}, x_{2}, x_{3}\right)=(1,-1,2)$ of the index-one cover $Q^{\sharp} \in Y^{\sharp}$ so that $\left.\left(x_{1}, x_{2}, x_{3}\right)\right|_{C^{\dagger}}=\left(t^{c / r_{Q}}, t^{1-c / r_{Q}}, 0\right)$. By $s_{C}(2)=-2$ and Lemmata 2.10] and 2.11] the map (2.7) has cokernel of length $\leq 1$ in Description 3.7)(1i) and $\leq 2$ in Description 3.7(ii) at $Q$. Hence we have the result by Lemma 2.12 except the value of $(H \cdot C)$. This value can be calculated from the equality $r s_{C}(-1)+r(E \cdot C)=-w_{Q}^{C}(1)$ obtained from the map $\mathcal{O}_{Y}(-E)^{\otimes r} \otimes \mathcal{O}_{Y}(r E) \otimes \mathcal{O}_{C} \rightarrow \mathcal{O}_{C}$.

Consider the case (ii). $w_{Q}^{C}(3)+w_{Q}^{C}(-3)=2 r_{Q}$. By $s_{C}(3)=-2$ and Lemmata 2.10 and 2.11 the map (2.7) has cokernel of length $\leq 1$ at $Q$. On the other hand, consider $x_{1}^{s_{1}} x_{2}^{s_{2}} x_{3}^{s_{3}}-x_{1}^{t_{1}} x_{2}^{t_{2}} x_{3}^{t_{3}}$ with $\left(s_{1}, s_{2}, s_{3}\right) \neq\left(t_{1}, t_{2}, t_{3}\right)$ such that $s_{1}-s_{2}+3 s_{3} \equiv$ $t_{1}-t_{2}+3 t_{3} \equiv 0$ modulo $r_{Q}$ and $a_{1} s_{1}+a_{2} s_{2}+a_{3} s_{3}=a_{1} t_{1}+a_{2} t_{2}+a_{3} t_{3}$. Then $\left(a_{1} s_{1}+a_{2} s_{2}+a_{3} s_{3}\right) / r_{Q} \geq 2$ for any such $x_{1}^{s_{1}} x_{2}^{s_{2}} x_{3}^{s_{3}}-x_{1}^{t_{1}} x_{2}^{t_{2}} x_{3}^{t_{3}}$, because $x_{1} x_{2}$ is the only invariant monomial whose restriction to $C^{\dagger}$ is $t$. Hence by the last paragraph in Section 2, the map (2.7) has cokernel of length $\geq 2+2-\left(a_{3}+w_{Q}^{C}(-3)\right) / r_{Q}=2$, which is a contradiction.

We need more accurate description developing Lemma 3.9 .

Lemma 3.10. Consider the case in Lemma 3.9 (i).

(i) If $f$ is of type IIb ${ }^{\vee \vee}$ in Description 3.7(1), we may choose the coordinates so that the restriction to $C^{\dagger}$ at one of $Q_{1}$ and $Q_{2}$ is of the form $\left(0, t^{c_{i}} / r_{i}\right.$, $\left.t^{1-\overline{a c_{i}} / r_{i}}\right)$ and that at the other is of the form $\left(t^{c_{i} / r_{i}}, 0, t^{\overline{a c_{i}} / r_{i}}\right)$.

(ii) If $f$ is of type III in Description [3.7(ii), we may choose the coordinates so that the restriction to $C^{\dagger}$ at $Q$ is of the form $\left(t^{c / r}, 0, t^{\overline{a c} / r}\right)$.

Proof. (ii) If both of them are of the form $\left(0, t^{c_{i} / r_{i}}, t^{1-\overline{a c_{i}} / r_{i}}\right)$, the image of the $\operatorname{map} \mathcal{O}_{Y}(-a E) \otimes \mathcal{O}_{Y}(E) \otimes \mathcal{O}_{Y}((a-1) E) \otimes \mathcal{O}_{C} \rightarrow \mathcal{O}_{C}$ is $\mathfrak{m}_{\left\{Q_{1}, Q_{2}\right\} \subset C \text {, whence }}$ $s_{C}(-a)+s_{C}(1)+s_{C}(a-1)=-2$. Thus $s_{C}(-a)=0$ by Lemma 3.2 (iii), and the statement follows from Lemma [3.8. On the other hand, if both are of the form $\left(t^{c_{i} / r_{i}}, 0, t^{\overline{{a c_{i}}_{i}} / r_{i}}\right)$, the image of the map $\mathcal{O}_{Y}(-a E) \otimes \mathcal{O}_{Y}(-E) \otimes \mathcal{O}_{Y}((a+1) E) \otimes$

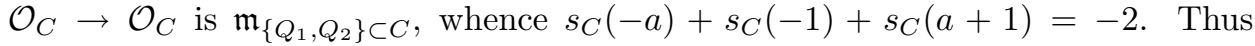
$s_{C}(-a)=0$ by $s_{C}(a+1) \leq-1$ and Lemma [3.2 (iii), and the statement follows from Lemma 3.8

(ii) If the restriction is of the form $\left(0, t^{c / r}, t^{1-\overline{a c} / r}\right)$, the image of the map $\mathcal{O}_{Y}(-a E) \otimes \mathcal{O}_{Y}(E) \otimes \mathcal{O}_{Y}((a-1) E) \otimes \mathcal{O}_{C} \rightarrow \mathcal{O}_{C}$ is $\mathfrak{m}_{Q \subset C}$, whence $s_{C}(-a)+$ 
$s_{C}(1)+s_{C}(a-1)=-1$. Thus $s_{C}(-a)=1$ by Lemma 3.2 (iv), and the statement follows from Lemma 3.8 .

Lemma 3.11. (i) Assume that $f$ is of type $I I b^{\vee \vee}$ with $s_{C}(-a)=-1$ in Description [3.7(1). Then $a<r_{1}<r_{2}, a_{11}=w_{Q_{1}}^{C}(1)=1, a_{22}=w_{Q_{2}}^{C}(-1)=$ 1 , and $(H \cdot C)=1 / r_{1}-1 / r_{2}$. In particular, for each $i$ we may choose semi-invariant local coordinates $x_{i 1}, x_{i 2}, x_{i 3}$ with weights $\operatorname{wt}\left(x_{i 1}, x_{i 2}, x_{i 3}\right)=$ $(1,-1, a)$ of the index-one cover $Q_{i}^{\sharp} \in Y^{\sharp}$ so that

$$
Q_{i}{ }^{\sharp} \in C^{\sharp} \subset Y^{\sharp} \cong o \in\left(x_{i i} \text {-axis }\right) \subset \mathbb{C}_{x_{i 1} x_{i 2} x_{i 3}}^{3} .
$$

(ii) Assume that $f$ is of type III with $s_{C}(-a)=0$ in Description [3.7](ii), and that $Q \in C$ has the local description in Lemma 3.9)(i). Then $a_{1}=w_{Q_{1}}^{C}(1)=$ 1 and $(H \cdot C)=1 / r$. In particular, we may choose semi-invariant local coordinates $x_{1}, x_{2}, x_{3}$ with weights $\operatorname{wt}\left(x_{1}, x_{2}, x_{3}\right)=(1,-1, a)$ of the indexone cover $Q^{\sharp} \in Y^{\sharp}$ so that

$$
Q^{\sharp} \in C^{\sharp} \subset Y^{\sharp} \cong o \in\left(x_{1} \text {-axis }\right) \subset \mathbb{C}_{x_{1} x_{2} x_{3}}^{3} .
$$

Proof. (ii) First of all, by Lemma 3.10(i) we can choose $(A, B)=(1,2)$ or $(2,1)$ so that the description at $Q_{A}$ is of the form $\left(t^{c_{A} / r_{A}}, 0, t^{\overline{a c_{A}} / r_{A}}\right)$ and that at $Q_{B}$ is of the form $\left(0, t^{c_{B} / r_{B}}, t^{1-\overline{a c_{B}} / r_{B}}\right)$. From the map $\mathcal{O}_{Y}(-E)^{\otimes r_{A} r_{B}} \otimes \mathcal{O}_{Y}\left(r_{A} r_{B} E\right) \otimes$ $\mathcal{O}_{C} \rightarrow \mathcal{O}_{C}$, we have $r_{A} r_{B} s_{C}(-1)+r_{A} r_{B}(E \cdot C)=-r_{B} w_{Q_{A}}^{C}(1)-r_{A} w_{Q_{B}}^{C}(1)$. By $w_{Q_{A}}^{C}(1)=c_{A}, w_{Q_{B}}^{C}(1)=r_{B}-c_{B}$ and $s_{C}(-1)=-1$ in Lemma 3.2(iii), we obtain

$$
1+(H \cdot C)=\frac{c_{A}}{r_{A}}+\frac{r_{B}-c_{B}}{r_{B}} .
$$

On the other hand, from the map $\mathcal{O}_{Y}(-a E)^{\otimes r_{A} r_{B}} \otimes \mathcal{O}_{Y}\left(a r_{A} r_{B} E\right) \otimes \mathcal{O}_{C} \rightarrow \mathcal{O}_{C}$ we have $r_{A} r_{B} s_{C}(-a)+a r_{A} r_{B}(E \cdot C)=-r_{B} w_{Q_{A}}^{C}(a)-r_{A} w_{Q_{B}}^{C}(a)$. By $w_{Q_{A}}^{C}(a)=\overline{a c_{A}}$, $w_{Q_{B}}^{C}(a)=r_{B}-\overline{a c_{B}}$ and $s_{C}(-a)=-1$, we obtain

$$
1+a(H \cdot C)=\frac{\overline{a c_{A}}}{r_{A}}+\frac{r_{B}-\overline{a c_{B}}}{r_{B}} .
$$

From the equalities (3.1) and (3.2), one of the inequalities $c_{A}<\overline{a c_{A}}$ and $c_{B}>\overline{a c_{B}}$ holds. However, $c_{B}>\overline{a c_{B}}$ cannot occur since $\left(r_{B}, 0\right)$ is contained in the semi-group (2.5) for $Q_{B}$. Hence $c_{A}<\overline{a c_{A}}$.

We claim that $c_{A}=1$. Suppose that $c_{A} \geq 2$, and take the smallest integer $m$ for which $c_{A} m \geq r_{A}$. $w_{Q_{A}}^{C}(m) \equiv c_{A} m-r_{A}$ modulo $r_{A}$ and $c_{A} m-r_{A}<a_{A 1}<a_{A 3}$. Thus the map $\mathcal{O}_{Y}(-m E) \otimes \mathcal{O}_{Y}(m E) \otimes \mathcal{O}_{C} \rightarrow \mathcal{O}_{C}$ at $Q_{A}$ is properly contained in $\mathfrak{m}_{Q_{A} \subset C}$. In our situation the image of the map $\mathcal{O}_{Y}(a E) \otimes \mathcal{O}_{Y}(-a E) \otimes \mathcal{O}_{C} \rightarrow \mathcal{O}_{C}$ is $\mathfrak{m}_{\left\{Q_{1}, Q_{2}\right\} \subset C}$. Considering also Lemma 3.2(iii), we have one of the following:

(i) $m>a$.

(ii) $r_{1} \mid m$ and $m<a$.

When (ii) occurs, $\overline{a c_{A}}=a c_{A}$. Then $c_{A}=1$ since $\left(r_{A}, 0\right)$ is contained in the semigroup (2.5) for $Q_{A}$. When (iii) occurs, unless $m=2$, the map $\mathcal{O}_{Y}(-(m-1) E) \otimes$ $\mathcal{O}_{Y}((m-1) E) \otimes \mathcal{O}_{C} \rightarrow \mathcal{O}_{C}$ at $Q_{A}$ is also properly contained in $\mathfrak{m}_{Q_{A} \subset C}$, which is a contradiction. The case where $m=2$ in (iii) remains. In this case, $r_{1}=2$ and $c_{1}=1$. Then we have $s_{C}(-a)=0$ as in the proof of Lemma 3.10] which contradicts the assumption $s_{C}(-a)=-1$. 
Now we have $(A, B)=(1,2)$ and $r_{1}<r_{2}$ by substituting $c_{A}=1$ in (3.1). We have

$$
\begin{aligned}
(H \cdot C) & =\frac{1}{r_{1}}-\frac{c_{2}}{r_{2}}, \\
a(H \cdot C) & =\frac{\bar{a}}{r_{1}}-\frac{\overline{a c_{2}}}{r_{2}} .
\end{aligned}
$$

In particular $\left\lfloor a / r_{1}\right\rfloor=\left\lfloor a c_{2} / r_{2}\right\rfloor$. We claim that $c_{2}=1$; then $a<r_{1}$ by Remark 2.1 and $(H \cdot C)=1 / r_{1}-1 / r_{2}$ by (3.3). This claim follows as in the proof of $c_{1}=1$, once we obtain $c_{2}<r_{2}-\overline{a c_{2}}$. But this inequality comes from the following equalities obtained by (3.3) and respectively (3.4):

$$
\begin{gathered}
1-(H \cdot C)=\frac{r_{1}-1}{r_{1}}+\frac{c_{2}}{r_{2}}, \\
1+a(H \cdot C)=\frac{\bar{a}}{r_{1}}+\frac{r_{2}-\overline{a c_{2}}}{r_{2}} .
\end{gathered}
$$

(iii) By Lemmata 3.9 and 3.10(iii), the description at $Q$ is of the form $\left(t^{c / r}, 0, t^{\overline{\bar{c}} / r}\right)$. From the map $\mathcal{O}_{Y}(-E)^{\otimes r} \otimes \mathcal{O}_{Y}(r E) \otimes \mathcal{O}_{C} \rightarrow \mathcal{O}_{C}$, we have $r s_{C}(-1)+r(E \cdot C)=$ $-w_{Q}^{C}(1)$. By $w_{Q}^{C}(1)=c$ and $s_{C}(-1)=0$ in Lemma 3.2(iv), we obtain $(H \cdot C)=c / r$. On the other hand, from the map $\mathcal{O}_{Y}(-a E)^{\otimes r} \otimes \mathcal{O}_{Y}(a r E) \otimes \mathcal{O}_{C} \rightarrow \mathcal{O}_{C}$, we have $r s_{C}(-a)+\operatorname{ar}(E \cdot C)=-w_{Q}^{C}(a)$. By $w_{Q}^{C}(a)=\overline{a c}$ and $s_{C}(-a)=0$, we have $a(H \cdot C)=\overline{a c} / r$. Hence $a c=\overline{a c}$ and the statement follows since $(r, 0)$ is contained in the semi-group (2.5).

The next lemma is an easy consequence by numerical argument.

Lemma 3.12. Assume that $f$ is of type $I I b^{\vee \vee}$. Then $(H \cap E)_{\text {red }}$ is irreducible.

Proof. We may assume that $s_{C}(-a)=-1$ by Lemma3.8. Write $[H \cap E]=u[C]+[F]$ cycle-theoretically, where the support of $[F]$ does not pass through $Q_{1}$ in Description 3.1(iii). Then we can write $(H \cdot[H \cap E])=u(H \cdot C)+v / r_{2}$. By $(H \cdot[H \cap E])=$ $E^{3}=a^{-1}\left(1 / r_{1}+1 / r_{2}\right)$ in Theorem 1.4 and $(H \cdot C)=1 / r_{1}-1 / r_{2}$ in Lemma3.11(ii), we obtain

$$
\frac{a u-1}{r_{1}}=\frac{a u+1-a v}{r_{2}} .
$$

Since $a \geq 2$ and $r_{1}<r_{2}$ by Lemma 3.11(i) , we have $v=0$.

We can take suitable surfaces by Lemma 3.4(1):

Description 3.13. $\quad$ (i) Assume that $f$ is of type $\mathrm{IIb}^{\vee V}$. Then by Lemma 3.411) and Remark 2.5)(1ii), there exist surfaces $S$ and $S_{0}$ on $Y$ defined by elements in $\mathcal{O}_{Y}(-a E)$ for which $(S \cap E)_{\text {red }} \nsubseteq(H \cap E)_{\text {red }} \cup\left(S_{0} \cap E\right)_{\text {red }}$. Let $D$ be an irreducible reduced curve in $S \cap E$ which intersects $H$ and $S_{0}$ properly. We can see that $D \cong \mathbb{P}^{1}$ as in Lemma 2.8

(ii) Assume that $f$ is of type III. Then by Lemma 3.4(1i) and Remark 2.5)(ii), we can take $H$ and $C \cong \mathbb{P}^{1} \subseteq H \cap E$ so that $C$ properly intersects the birational transform $H_{0}$ of a general hyperplane section on $P \in X$.

Lemma 3.14. Assume that $f$ is of type $I I b^{\vee \vee}$ with $s_{C}(-a)=-1$, and take $C \subseteq$ $H \cap E$ and $D \subseteq S \cap E$ as in Descriptions 3.7 (i) and 3.13(i). Then we can write $[S \cap E]=x[C]+[D]$ cycle-theoretically for some positive integer $x$, and $D$ intersects $C$ exactly at one of $Q_{1}$ and $Q_{2}$. Let $Q_{i}$ with $i=1$ or 2 be the one which $D$ passes 
through. Then $(H \cdot D)=1 / r_{i}$, and we may choose semi-invariant local coordinates $x_{1}, x_{2}, x_{3}$ with weights wt $\left(x_{1}, x_{2}, x_{3}\right)=(1,-1, a)$ of the index-one cover $Q_{i}^{\sharp} \in Y^{\sharp}$ so that

$$
Q_{i}^{\sharp} \in D^{\sharp} \subset Y^{\sharp} \cong o \in\left(x_{1} \text {-axis }\right) \subset \mathbb{C}_{x_{1} x_{2} x_{3}}^{3} .
$$

Proof. Write $[S \cap E]=x[C]+\left[D^{\prime}\right]$ cycle-theoretically. We can write $1 / r_{1}+1 / r_{2}=$ $(H \cdot[S \cap E])=x(H \cdot C)+y / r_{1}+z / r_{2}$. If $y=z=1$, then $[S \cap E]=\left[D^{\prime}\right]$ and $(S \cdot C) \geq 1 / r_{1}+1 / r_{2}$ since $S$ passes through $Q_{1}$ and $Q_{2}$. On the other hand, $(S \cdot C) \leq(S \cdot[H \cap E])=1 / r_{1}+1 / r_{2}$ by Theorem 1.4. Hence $C=H \cap E$ and $s_{C}(-a) \geq 0$ by Lemma 3.4 (iii) with $i=a$, with Remark 2.5(ii) and Lemma 3.11(i). It contradicts $s_{C}(-a)=-1$.

Hence $y=0$ or $(y, z)=(1,0)$. By $(H \cdot[S \cap E])=1 / r_{1}+1 / r_{2}$ and $(H \cdot C)=$ $1 / r_{1}-1 / r_{2}$ in Lemma 3.11(ii), we obtain

$$
\frac{x-1+y}{r_{1}}=\frac{x+1-z}{r_{2}} .
$$

Because $y=0$ or $(y, z)=(1,0)$, and $r_{1}<r_{2}$ in Lemma 3.111i), we have $(y, z)=$ $(1,0)$ or $(0,1)$. Therefore, provided $r_{1} \nmid r_{2}, D^{\prime}=D$ and $D$ intersects $C$ only at $Q_{1}$ or $Q_{2}$; then $(H \cdot D)=1 / r_{1}$, respectively $1 / r_{2}$. However, if $r_{1} \mid r_{2}$ and $\left(H \cdot D^{\prime}\right)=1 / r_{1}$, then setting $\left(r_{1}, r_{2}\right)=(r, s r)$, by Lemma 3.12 we can write

$$
a u\left(\frac{1}{r}-\frac{1}{s r}\right)=\frac{1}{r}+\frac{1}{s r},
$$

where $[H \cap E]=u[C]$ for some $u \geq 2$. By this equality and $s>1$ in Lemma3.11(ii), we have $(s, a u)=(2,3)$ or $(3,2)$. But this contradicts $a \geq 2$ and $u \geq 2$.

Let $Q_{i}$ with $i=1$ or 2 be the one which $D$ passes through. From the map $\mathcal{O}_{Y}(-E)^{\otimes r_{i}} \otimes \mathcal{O}_{Y}\left(r_{i} E\right) \otimes \mathcal{O}_{D} \rightarrow \mathcal{O}_{D}$, we obtain $r_{i} s_{D}(-1)+r_{i}(E \cdot D)=-w_{Q_{i}}^{D}(1)$. Hence $w_{Q_{i}}^{D}(1) \equiv 1$ modulo $r_{i}$. Therefore $w_{Q_{i}}^{D}(1)=1$, since $\left(r_{i}, 0\right)$ is contained in the semi-group (2.5) for $Q_{i} \in D$. Thus the desired description follows.

The next theorem gives complete description in types IIb ${ }^{\vee \vee}$ and III:

Theorem 3.15. Assume that $f$ is of type II ${ }^{\vee \vee}$ and III. Then there exist a surface $H$ and a curve $C \subseteq H \cap E$ in Description 3.7 which satisfy the assumption in Lemma 3.8 .

Proof. We first treat type $\mathrm{IIb}^{\vee \vee}$. Suppose that $C$ does not satisfy the assumption in Lemma 3.8, that is, suppose that $s_{C}(-a)=-1$ by Lemma 3.2(iii). Then by Description 3.13(1i) and Lemma 3.14, there exist $S$ and $Q_{i} \in D \cong \mathbb{P}^{1}$ for which we may choose semi-invariant local coordinates $x_{1}, x_{2}, x_{3}$ so that $\left.\left(x_{1}, x_{2}, x_{3}\right)\right|_{D^{\dagger}}=$ $\left(t^{1 / r_{i}}, 0,0\right)$. $D$ intersects $H$ properly.

Let $H^{\sharp}$ be the pre-image of $H$ on the index-one cover $Q_{i}^{\sharp} \in Y^{\sharp}$. Write $h\left(x_{1}\right)+$ $g_{1} x_{2}+g_{2} x_{3}$ for the defining function of $H^{\sharp}$ at $Q_{i}^{\sharp}$, where $h\left(x_{1}\right)$ is a semi-invariant formal series of weight 1 and $g_{1}, g_{2}$ are semi-invariant formal series in $x_{1}, x_{2}, x_{3}$. The order of the function for $\left.H^{\sharp}\right|_{D^{\dagger}}$ with respect to $t$ equals $(H \cdot D)=1 / r_{i}$ by Lemma 3.14 It means that $h\left(t^{1 / r_{i}}\right)$ has order $1 / r_{i}$ with respect to $t$, whence the monomial $x_{1}$ appears in $h$ with non-zero coefficient. Thus we may choose the coordinates $x_{1}, x_{2}, x_{3}$ so that $H^{\sharp}$ is given by $x_{1}=0$. If $Q_{i}=Q_{1}$, for the coordinates $x_{11}, x_{12}, x_{13}$ in Lemma3.11(1) we can write $x_{1}=c x_{11}+g\left(x_{11}, x_{12}, x_{13}\right)$, where $c \neq 0$ and $g$ is a semi-invariant formal series of weight 1 in which the monomial $x_{11}$ does 
not appear. Thus $\left.x_{1}\right|_{C^{\dagger}} \neq 0$ by $\left.\left(x_{11}, x_{12}, x_{13}\right)\right|_{C^{\dagger}}=\left(t^{1 / r_{1}}, 0,0\right)$, which contradicts $C \subset H$.

Therefore $Q_{i}=Q_{2}$. We consider a general $S_{0}$ in Description 3.13)(i). $S_{0} \cap E$ also has a component $D_{0} \cong \mathbb{P}^{1}$ which has the same properties as $D \subset S \cap E$ has, and we can choose new coordinates $x_{1}{ }^{\prime}, x_{2}{ }^{\prime}, x_{3}{ }^{\prime}$ and $t^{\prime}$ for $Q_{2} \in D_{0} \subset Y$ so that $\left.\left(x_{1}{ }^{\prime}, x_{2}{ }^{\prime}, x_{3}{ }^{\prime}\right)\right|_{D_{0}^{\dagger}}=\left(t^{\prime 1 / r_{2}}, 0,0\right)$. Since $\left(S \cdot D_{0}\right)=a\left(H \cdot D_{0}\right)=a / r_{2}<$ 1 by Lemma 3.14 and Remark 2.1, $S$ intersects $D_{0}$ only at $Q_{2}$. Let $S^{\sharp}$ be the pre-image of $S$ on $Y^{\sharp}$, and $g\left(x_{1}, x_{2}, x_{3}\right)=h\left(x_{1}{ }^{\prime}, x_{2}{ }^{\prime}, x_{3}{ }^{\prime}\right)$ the defining function of $S^{\sharp}$ at $Q_{2}^{\sharp}$. $h\left(t^{\prime 1 / r_{2}}, 0,0\right)$, the function for $\left.S^{\sharp}\right|_{D_{0}^{\dagger}}$, has order $\left(S \cdot D_{0}\right)=a / r_{2}$ with respect to $t$, whence we can write $h=\left(c^{\prime}+h_{1}\left(x_{1}{ }^{\prime}\right) x_{1}^{\prime}{ }^{r_{2}}\right) x_{1}^{\prime}{ }^{a}+h_{2}\left(x_{1}{ }^{\prime}, x_{2}{ }^{\prime}, x_{3}{ }^{\prime}\right) x_{2}{ }^{\prime}+$ $h_{3}\left(x_{1}{ }^{\prime}, x_{2}{ }^{\prime}, x_{3}{ }^{\prime}\right) x_{3}{ }^{\prime}$, where $c^{\prime} \neq 0$ and $h_{1}, h_{2}, h_{3}$ are semi-invariant with weights $0, a+1,0$. Thus we can write $g=c x_{1}^{a}+\cdots$ for some $c \neq 0$, and we see that $g\left(t^{1 / r_{2}}, 0,0\right)=0$ holds only if $h_{3}$ is a unit, by considering weights and $a<r_{1}<r_{2}$ in Description 3.13(ii). Of course $g\left(t^{1 / r_{2}}, 0,0\right)=0$ by $D \subset S$, whence $h_{3}$ is a unit and $S^{\sharp}$ is smooth at $Q_{2}^{\sharp}$.

Hence we may choose the coordinates $x_{21}, x_{22}, x_{23}$ in Lemma 3.11(1i) so that $x_{21}=x_{1}$, which is the function for $H^{\sharp}$, and so that $x_{23}$ is the function for $S^{\sharp}$. In particular the scheme $S \cap H$ is irreducible and reduced at the generic point of $C$. Write $[H \cap E]=u[C]$ by Lemma 3.12 and $[S \cap E]=x[C]+[D]$ by Lemma 3.14 Then we have

$$
\begin{aligned}
u a\left(\frac{1}{r_{1}}-\frac{1}{r_{2}}\right) & =\frac{1}{r_{1}}+\frac{1}{r_{2}}, \\
x\left(\frac{1}{r_{1}}-\frac{1}{r_{2}}\right)+\frac{1}{r_{2}} & =\frac{1}{r_{1}}+\frac{1}{r_{2}} .
\end{aligned}
$$

Thus $(u a-x)\left(r_{2}-r_{1}\right)=r_{1}$ and we can write $r_{1}=n r, r_{2}=(n+1) r$. Note that

$$
\begin{gathered}
u a=2 n+1, \\
x=n+1 .
\end{gathered}
$$

On the other hand, let $\pi: \hat{E} \rightarrow E$ be the normalisation of $E$ and set $\hat{C}:=$ $\left(C \times_{E} \hat{E}\right)_{\text {red }}$. The coefficients of $[C]$ in the 1-cycles $[S \cap E]$ and $[a H \cap E]$ are determined by the lengths of the schemes $\pi^{*} S \cap \hat{C}$ and $\pi^{*}(a H) \cap \hat{C}$ at all the generic points of $\hat{C}$. From this point of view, we see that $(S \cap E) \cap(a H \cap E)$ contains the 1-cycle $x[C]$ because of the general choice of $S$. Here $(S \cap E) \cap(a H \cap E) \subseteq S \cap a H$, and $S \cap H$ is irreducible and reduced at the generic point of $C$. Hence $x \leq a$, but it contradicts (3.5), (3.6) and $u \geq 2$.

Now we treat type III. We start with $H, Q \in C$ and $H_{0}$ in Description 3.13)(ii). Suppose that $C$ does not satisfy the assumption in Lemma 3.8 that is, suppose that $s_{C}(-a)=0$. Then by Lemmata 3.9 and 3.11)(ii), we may choose coordinates $x_{1}, x_{2}, x_{3}$ so that $\left.\left(x_{1}, x_{2}, x_{3}\right)\right|_{C^{\dagger}}=\left(t^{1 / r}, 0,0\right),\left(t^{2 / r}, t^{1-2 / r}, 0\right)$ or $\left(t^{1-2 / r}, t^{2 / r}, 0\right)$. Note that $\left(H_{0} \cdot E\right)=1 / r, 2 / r, 1-2 / r$ respectively in these cases. Thus $H_{0}$ intersects $C$ only at $Q$. Let $H_{0}^{\sharp}$ be the pre-image of $H_{0}$ on the index-one cover $Q^{\sharp} \in Y^{\sharp}$, and $g\left(x_{1}, x_{2}, x_{3}\right)$ the defining function of $H_{0}^{\sharp}$ at $Q^{\sharp}$. Then $g$ is semiinvariant of weight 1 and $g\left(t^{1 / r}, 0,0\right), g\left(t^{2 / r}, t^{1-2 / r}, 0\right)$ and $g\left(t^{1-2 / r}, t^{2 / r}, 0\right)$ have order $1 / r, 2 / r, 1-2 / r$ with respect to $t$. As in the former argument, we can 
see that the monomial $x_{1}$ appears in the function for $H_{0}^{\sharp}$ with non-zero coefficient in each of the cases. Hence we may choose semi-invariant local coordinates $x_{1}, x_{2}, x_{3}$ with weights $\operatorname{wt}\left(x_{1}, x_{2}, x_{3}\right)=(1,-1, a)$ of $Q^{\sharp} \in Y^{\sharp}$ so that $H_{0}$ is given by $x_{1}=0$. We take $Q \in C_{0} \cong \mathbb{P}^{1} \subset H_{0} \cap E$. If $s_{C_{0}}(-a) \neq 1$, then $s_{C_{0}}(-a)=0$ and we may choose new coordinates $x_{1}{ }^{\prime}, x_{2}{ }^{\prime}, x_{3}{ }^{\prime}$ and $t^{\prime}$ so that $\left.\left(x_{1}{ }^{\prime}, x_{2}{ }^{\prime}, x_{3}{ }^{\prime}\right)\right|_{C_{0}^{\dagger}}=\left(t^{\prime 1 / r}, 0,0\right),\left(t^{\prime 2 / r}, t^{\prime 1-2 / r}, 0\right)$ or $\left(t^{1-2 / r}, t^{\prime 2 / r}, 0\right)$. On the other hand, we can write $x_{1}=c x_{1}{ }^{\prime}+g\left(x_{1}{ }^{\prime}, x_{2}{ }^{\prime}, x_{3}{ }^{\prime}\right)$, where $c \neq 0$ and $g$ is a semi-invariant formal series of weight 1 in which the monomial $x_{1}^{\prime}$ does not appear. Thus $\left.x_{1}\right|_{C_{0}^{\dagger}} \neq 0$, which contradicts $C_{0} \subset H_{0}$.

\section{Existence of Du Val sections}

In this section we prove Theorem 1.7 the general elephant theorem. It was proved when $f$ is of type $\mathrm{O}$ or $\mathrm{I}$ in Theorem 2.7, and is trivial in type IV. Thus we keep the assumption that $f$ is of type II or III. First we restate the results in Section 3

Description 4.1. (i) (type IIa; Theorem 3.5)

(a) $a=2$ and $[H \cap E]=\left[\mathbb{P}^{1}\right]+\left[\mathbb{P}^{1}\right] ; s_{C}(-2)=0$ and $(H \cdot C)=1 / r ; Q \in C$ is locally expressed by semi-invariant local coordinates $x_{1}, x_{2}, x_{3}$ with weights $\operatorname{wt}\left(x_{1}, x_{2}, x_{3}\right)=(1,-1,4)$ of the index-one cover $Q^{\sharp} \in Y^{\sharp}$ as $\left.\left(x_{1}, x_{2}, x_{3}\right)\right|_{C^{\dagger}}=\left(t^{(r+1) / 2 r}, 0, t^{2 / r}\right)$ when $r \equiv 1$ modulo 4 , and $\left(0, t^{(r-1) / 2 r}, t^{2 / r}\right)$ when $r \equiv 3$ modulo 4 .

(b) $r=5, a=4$ and $H \cap E=\mathbb{P}^{1} ; s_{C}(-4)=0$ and $(H \cdot C)=1 / r ; Q \in C$ is locally expressed by semi-invariant local coordinates $x_{1}, x_{2}, x_{3}$ with weights $\operatorname{wt}\left(x_{1}, x_{2}, x_{3}\right)=(1,-1,3)$ of the index-one cover $Q^{\sharp} \in Y^{\sharp}$ as $\left.\left(x_{1}, x_{2}, x_{3}\right)\right|_{C^{\dagger}}=\left(t^{3 / 5}, t^{2 / 5}, 0\right)$.

(ii) (type $\mathrm{IIb}^{\vee}$; Theorem 3.6$)$

(a) $J=\{(r, 1),(r, 1)\}, a=2$ and $H \cap E=\mathbb{P}^{1} ; s_{C}(-2)=0$ and $(H \cdot C)=1 / r ; Q \in C$ is locally expressed by semi-invariant local coordinates $x_{1}, x_{2}, x_{3}, x_{4}$ with weights $\operatorname{wt}\left(x_{1}, x_{2}, x_{3}, x_{4}\right)=(1,-1,2,0)$ of the index-one cover $Q^{\sharp} \in Y^{\sharp}$ as $\left.\left(x_{1}, x_{2}, x_{3}, x_{4}\right)\right|_{C^{\dagger}}=\left(0,0, t^{2 / r}, t\right)$.

(b) $J=\{(2,1),(4,1)\}, a=3$ and $H \cap E=\mathbb{P}^{1} ; s_{C}(-3)=0$ and $(H \cdot C)=$ $1 / 4 ; Q \in C$ is locally expressed by semi-invariant local coordinates $x_{1}, x_{2}, x_{3}, x_{4}$ with weights wt $\left(x_{1}, x_{2}, x_{3}, x_{4}\right)=(1,3,3,2)$ of the indexone cover $Q^{\sharp} \in Y^{\sharp}$ as $\left.\left(x_{1}, x_{2}, x_{3}, x_{4}\right)\right|_{C^{\dagger}}=\left(0,0, t^{3 / 4}, t^{1 / 2}\right)$.

(iii) (type IIb ${ }^{\vee}$; Theorem 3.15) $H \cap E=\mathbb{P}^{1} ; s_{C}(-a)=0$ and $(H \cdot C)=\left(r_{1}+\right.$ $\left.r_{2}\right) / a r_{1} r_{2} ; Q_{i} \in C$ is locally expressed by semi-invariant local coordinates $x_{i 1}, x_{i 2}, x_{i 3}$ with weights wt $\left(x_{i 1}, x_{i 2}, x_{i 3}\right)=(1,-1, a)$ of the index-one cover $Q_{i}^{\sharp} \in Y^{\sharp}$ as $\left.\left(x_{i 1}, x_{i 2}, x_{i 3}\right)\right|_{C^{\dagger}}=\left(0,0, t^{1 / r_{i}}\right)$.

(iv) (type III; Theorem 3.15) We can choose $H$ so that $H \cap E=\mathbb{P}^{1} ; s_{C}(-a)=1$ and $(H \cdot C)=(r+1) / a r ; Q \in C$ is locally expressed by semi-invariant local coordinates $x_{1}, x_{2}, x_{3}$ with weights wt $\left(x_{1}, x_{2}, x_{3}\right)=(1,-1, a)$ of the index-one cover $Q^{\sharp} \in Y^{\sharp}$ as $\left.\left(x_{1}, x_{2}, x_{3}\right)\right|_{C^{\dagger}}=\left(0,0, t^{1 / r}\right)$.

Theorem 4.2. Let $S$ be a general elephant of $Y$.

(i) If $f$ is of type II, then $S$ intersects $H \cap E$ at and only at the non-Gorenstein points of $Y$.

(ii) If $f$ is of type III, then $S$ intersects $H \cap E$ at and only at a general point of $C$ and the non-Gorenstein point of $Y$. 
Proof. (1i) Take any $C \cong \mathbb{P}^{1} \subseteq H \cap E$. By (2.3), Lemma 4.3 and the commutative diagrams in Section 2], we obtain a surjective map $f_{*} \mathcal{O}_{Y}(-a E) \rightarrow H^{0}\left(\left[\mathcal{O}_{Y}(-a E)\right]_{C}\right)$. Recalling that $s_{C}(-a)=0$ in Description 4.1, we have $H^{0}\left(\left[\mathcal{O}_{Y}(-a E)\right]_{C}\right)=\mathbb{C}$. Hence we find a general elephant $S$ which properly intersects $H \cap E$. Of course $S$ passes through any non-Gorenstein point of $Y$. On the other hand, because $(S \cdot[H \cap E])=a E^{3} \leq 1$ in any case by Description 4.1 we see that $S$ does not intersect $H \cap E$ at any other point.

(ii) It suffices to consider $\left[\mathcal{O}_{Y}(-a E) \otimes \mathfrak{m}_{Q^{\prime} \subset Y}\right]_{C}$ instead of $\left[\mathcal{O}_{Y}(-a E)\right]_{C}$ since $s_{C}(-a)=1$ in Description 4.1 .

Lemma 4.3. Assume that $f$ is of type IIa with $a=2$. Write $[H \cap E]=[C]+\left[C^{\prime}\right]$ cycle-theoretically. Then there exists a natural exact sequence

$$
0 \rightarrow \mathcal{O}_{C^{\prime}}(-1) \rightarrow \mathcal{O}_{H \cap E} \rightarrow \mathcal{O}_{C} \rightarrow 0 .
$$

Proof. Let $\mathcal{I}$ be the kernel of the natural map $\mathcal{O}_{H \cap E} \rightarrow \mathcal{O}_{C}$. $\mathcal{I}$ is supported on $C^{\prime} \cong \mathbb{P}^{1}$. By $H^{0}\left(\mathcal{O}_{H \cap E}\right)=\mathbb{C}$ in Remark [2.9, we have $H^{0}(\mathcal{I})=0$. By $H^{1}\left(\mathcal{O}_{H \cap E}\right)=$ 0 in Lemma 2.8)(iii), we have $H^{1}(\mathcal{I})=0$. Hence $\mathcal{I} \cong \mathcal{O}_{\mathbb{P}^{1}}(-1)$.

It is the time to prove Theorem [1.7, the main theorem.

Theorem 4.4. Assume that $f$ is of type II or III. Then a general elephant $S$ of $Y$ has at worst $D u$ Val singularities. Moreover, the type of any Du Val singularity $Q \in S$ is the same as that of a general elephant of a germ $Q \in Y$.

Proof. By [7] it suffices to consider the case where $P$ is a singular point. By Theorem 4.2 and the theorem of Bertini, it is enough to show that $S$ has a Du Val singularity at each non-Gorenstein point $Q \in Y$ the type of which is the same as that of a general elephant of a germ $Q \in Y$.

First we treat type IIa in Description 4.1(1). Let $Q \in C=H \cap E$ be the nonGorenstein point and a curve which are investigated.

Consider the case $a=2$. It is enough to show that the monomial $x_{3}$ appears in the defining function $h\left(x_{1}, x_{2}, x_{3}\right)$ of $S$ in Theorem 4.2 with non-zero coefficient. Since $S$ intersects $C$ only at $Q$ by Theorem $4.2 h\left(t^{(r+1) / 2 r}, 0, t^{2 / r}\right)$ when $r \equiv 1$ modulo 4 , and $h\left(0, t^{(r-1) / 2 r}, t^{2 / r}\right)$ when $r \equiv 3$ modulo 4 , have order $(S \cdot C)=2 / r$ with respect to $t$. Hence the monomial $x_{3}$ appears in $h$ with non-zero coefficient.

Consider the case $a=4$. Then $-K_{Y}$ is linearly equivalent to $E+5 H$. Thus it is enough to show that

(i) $E$ has a Du Val singularity of type $A_{4}$ at $Q$, and

(ii) $|5 H|$ is free at $Q$.

We first see (1]. By [14] we can consider a birational morphism $g: Z \rightarrow Y$ such that $Z$ has a $g$-exceptional divisor $F$ the discrepancy of which with respect to $K_{X}$ is 1 . Write

$$
\begin{aligned}
K_{Z} & =g^{*} K_{Y}+b F+(\text { others }), \\
g^{*} E & =E_{Z}+m F+(\text { others }),
\end{aligned}
$$

where $E_{Z}$ is the birational transform of $E$. Then $K_{Z}=g^{*}\left(f^{*} K_{X}+4 E\right)+b F+$ (others) $=g^{*} f^{*} K_{X}+4 E_{Z}+(b+4 m) F+$ (others), whence $b=m=1 / 5$ since $5 b, 5 m \in \mathbb{Z}$. Thus the defining function of $E$ in $x_{1}, x_{2}, x_{3}$ in Description 4.1(i) has a non-zero linear term. Considering the weight, we see that the monomial $x_{3}$ appears 
in the function for $E$ with non-zero coefficient. Hence we may take $x_{3}$ so that $E$ is given by $x_{3}=0$. This implies (i).

It is easy to see (iii). From (2.3) and the commutative diagrams in Section 2 we obtain a surjective map $f_{*} \mathcal{O}_{Y}(-5 E) \rightarrow H^{0}\left(\left[\mathcal{O}_{Y}(-5 E)\right]_{C}\right)=H^{0}\left(\mathcal{O}_{\mathbb{P}^{1}}(1)\right)$, which shows (ii).

Secondly we treat type $\mathrm{IIb}^{\vee}$ in Description 4.1(iii). Let $Q \in C=H \cap E$ be the non-Gorenstein point and a curve which are investigated. $Q \in Y$ is described as in Remark 1.5. Consider a general elephant $T$ on a germ $Q \in Y$, for which the function in $y_{1}, y_{2}, y_{3}, y_{4}$ has a non-zero linear term. Then by Remark 1.5 we have the following:

(i) If $J=\{(r, 1),(r, 1)\}$ and $r \geq 5$, the linear term of the function for $T$ is $y_{3}$. Any such $T$ gives a Du Val singularity of type $A_{2 r-1}$, which is the type of a general elephant of a germ $Q \in Y$.

(ii) In any other case, the linear term of the function for $T$ is a linear combination of $y_{2}$ or $y_{3}$. Any $T$ which has a general linear term gives a Du Val singularity of type $A_{5}, E_{6}$ or $D_{5}$ according to (ia), (ib), (ii) in Remark 1.5 respectively, which is the type of a general elephant of a germ $Q \in Y$.

Thus if $J=\{(r, 1),(r, 1)\}$ and $r \geq 5$, it is enough to show the following:

Claim 4.4.1. The function for $S$ in Theorem 4.2 in $y_{1}, y_{2}, y_{3}, y_{4}$ has a non-zero linear term.

In any other case, $-K_{Y}$ is linearly equivalent to $E+r H$. Thus it is enough to show, adding to Claim 4.4.1

Claim 4.4.2. (i) The function for $E$ in $y_{1}, y_{2}, y_{3}, y_{4}$ has a non-zero linear term which is linearly independent to that in the function for $S$.

(ii) $|r H|$ is free at $Q$.

However, in Claim 4.4.2, the existence of a non-zero linear term in the function for $E$ and Claim 4.4.2(ii) follow as in the proof of type IIa with $a=4$. In particular, in Claim 4.4.2 we may choose coordinates $x_{1}, x_{2}, x_{3}, x_{4}$ in Description 4.1(ii) so that $E$ is given by $x_{2}=0$. Hence Claims 4.4.1 and 4.4 .2 follow if we prove that the monomial $x_{3}$ appears in the defining function $h\left(x_{1}, x_{2}, x_{3}, x_{4}\right)$ of $S$ in Theorem 4.2 with non-zero coefficient. Because $S$ intersects $C$ only at $Q$ by Theorem 4.2 $h\left(0,0, t^{2 / r}, t\right)$ and $h\left(0,0, t^{3 / 4}, t^{1 / 2}\right)$ have order $(S \cdot C)=a E^{3}=2 / r$, respectively $3 / 4$ with respect to $t$, according to $J=\{(r, 1),(r, 1)\}$, respectively $\{(2,1),(4,1)\}$. Hence the monomial $x_{3}$ appears in $h$ with non-zero coefficient.

Finally we treat types IIb ${ }^{\vee}$ and III in Description 4.1(iii), (iv). Let $Q \in C=$ $H \cap E$ be a non-Gorenstein point and a curve which are investigated. It is enough to show that $x_{3}$ appears in the defining equation $h\left(x_{1}, x_{2}, x_{3}\right)$ of $S$ in Theorem 4.2 with non-zero coefficient. We can see this similarly, remarking that the local intersection number of $S$ and $C$ at $Q$ is $1 / r_{Q}$ by Description 4.1(iii), (iv) and Theorem 4.2.

\section{Possible types of Singularities}

In this section we restrict the types of a general elephant $S$ of $Y$ and its birational transform $S_{X}$ on $X$, and deduce Theorem 1.8. First we recall the dual graphs for minimal resolutions of Du Val singularities. o denotes an exceptional curve and - the birational transform of a general hyperplane section, and each exceptional curves $F_{i}$ is marked with its coefficient in the fundamental cycle. 
(i) (type $A_{n}$ )

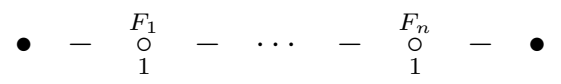

(ii) (type $D_{n}$ )

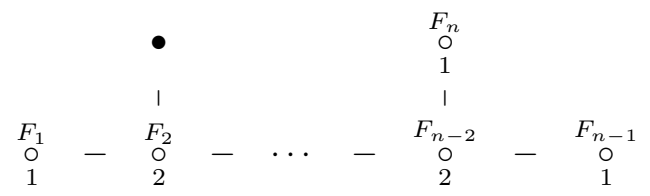

(iii) (type $E_{6}$ )

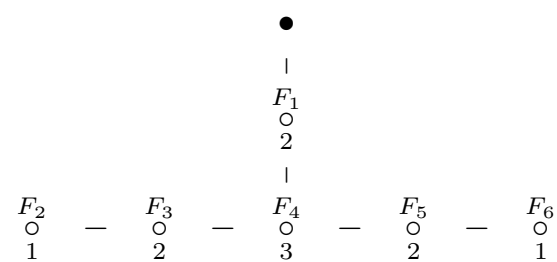

(iv) (type $E_{7}$ )

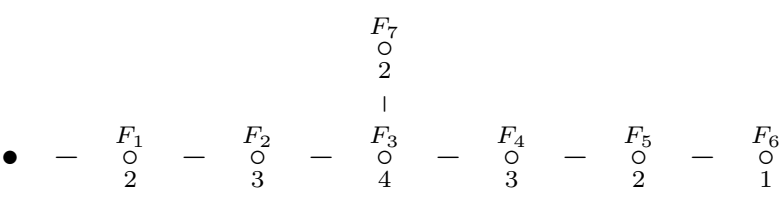

(v) (type $E_{8}$ )

$$
\begin{gathered}
F_{8} \\
0 \\
3 \\
1 \\
0 \\
1 \\
0
\end{gathered}
$$

Take a general elephant $S$ of $Y$, and write $S_{X}$ for its birational transform on $X$. A general hyperplane section $P \in H_{X}$ on $X$ gives also a general hyperplane section $P \in D_{X}:=\left.H_{X}\right|_{S_{X}}$ on $S_{X}$. Let $f_{S}: S \rightarrow S_{X}$ be the induced map. Since $S$ and $S_{X}$ have at worst $\mathrm{Du}$ Val singularities by Theorem 1.7, $f_{S}$ factors through the minimal resolution of $S_{X}$. Let $Z$ be the birational transform on $S$ of the fundamental cycle. Then $f^{*} H_{X}=H+b E$ as in Section 2 and $f^{*} D_{X}=D+Z=\left.H\right|_{S}+\left.b E\right|_{S}$, where $D$ is the birational transform on $S$ of $D_{X}$. Note that $\left.b E\right|_{S} \leq Z$. We also have

$$
l:=\left(\left.\left.H\right|_{S} \cdot b E\right|_{S}\right)=b^{2} a E^{3} .
$$

Assume that $f$ is of type I. In this case, $a=b=2$ and either $J=\{(7,3)\}$ with $E^{3}=1 / 7$ or $J=\{(3,1),(5,2)\}$ with $E^{3}=1 / 15$ holds by Theorems 1.4 and 2.7 and Proposition 2.4

Lemma 5.1. Assume that $f$ is of type I. Take a general elephant $S$ of $Y$.

(i) If $J=\{(7,3)\}$, the dual graph for the partial resolution $S \rightarrow S_{X}$ is

$$
*-\underset{2}{\circ} \text { or } \quad \stackrel{\circ}{2}-*-\underset{2}{\circ}
$$

where the attached numbers are the coefficients in $\left.2 E\right|_{S}$, and $*$ means that a Du Val singularity of type $A_{6}$ or worse appears there. 
(ii) If $J=\{(3,1),(5,2)\}$, the dual graph for the partial resolution $S \rightarrow S_{X}$ is

$$
*-\underset{2}{\circ}-\star \text { or } *-\underset{4}{\circ}-\star,
$$

where the attached number is the coefficient in $\left.2 E\right|_{S}$, and $*$ and $\star$ mean that a Du Val singularity of type $A_{2}$, respectively $A_{4}$, or worse appears there.

Proof. (1i) Since $(-E \cdot[S \cap E])=2 E^{3}=2 / 7$, it is enough to show the existence of $S$ such that $S \cap E$ defines a reduced 1-cycle. We can calculate $\chi\left(\mathcal{Q}_{-2}\right)=2$ by (2.2). Thus we have the desired $S$ as in the proof of Lemma 3.4(ii).

(iii) Since $(-E \cdot[S \cap E])=2 E^{3}=2 / 15$, it is enough to show the existence of $S$ such that $S \cap E$ defines an irreducible but possibly non-reduced 1-cycle. By $(-E \cdot[S \cap E])<1 / 5$, each irreducible component of $(S \cap E)_{\text {red }}$ passes through all the non-Gorenstein points of $Y$. Hence $(S \cap E)_{\text {red }}=\mathbb{P}^{1}$ by $h^{1}\left(\mathcal{O}_{S \cap E}\right)=0$ in Remark 2.9]

We restrict the dual graph for the partial resolution $S$, considering $\left.2 E\right|_{S} \leq Z$ and Lemma 5.1 The following table shows all the possibilities up to permutation in terms of $F_{i}$ :

\begin{tabular}{l|l|l|l||l|l|l|l}
\hline \multicolumn{3}{c||}{$J=\{(7,3)\}$} & \multicolumn{4}{c}{$J=\{(3,1),(5,2)\}$} \\
\hline$S_{X}$ & \multicolumn{1}{|c|}{$Z$} & \multicolumn{1}{c|}{$2 E_{S}$} & \multicolumn{1}{c||}{$l$} & $S_{X}$ & \multicolumn{1}{c}{$Z$} & $2 E_{S}$ & \multicolumn{1}{c}{$l$} \\
\hline$D_{n}$ & $2 F_{m}+\cdots$ & $2 F_{m}+\cdots$ & $4 / m$ & $D_{n}$ & $2 F_{m}$ & $2 F_{m}$ & $4 / m$ \\
$E_{7}$ & $2 F_{1}+\cdots$ & $2 F_{1}+\cdots$ & 2 & $E_{7}$ & $3 F_{4}$ & $2 F_{4}$ & $8 / 15$ \\
& $2 F_{5}$ & $2 F_{5}$ & 1 & $E_{8}$ & $4 F_{3}$ & $2 F_{3}$ & $1 / 3$ \\
& $2 F_{7}$ & $2 F_{7}$ & $8 / 7$ & & & $4 F_{3}$ & $4 / 3$ \\
$E_{8}$ & $2 F_{1}+\cdots$ & $2 F_{1}+\cdots$ & 2 & & $5 F_{4}$ & $2 F_{4}$ & $1 / 5$ \\
& $3 F_{2}$ & $2 F_{2}$ & $2 / 3$ & & & $4 F_{4}$ & $4 / 5$ \\
& $3 F_{2}+2 F_{7}$ & $2 F_{2}+2 F_{7}$ & 1 & & $6 F_{5}$ & $2 F_{5}$ & $2 / 15$ \\
& $4 F_{3}$ & $2 F_{3}$ & $1 / 3$ & & & $4 F_{5}$ & $8 / 15$ \\
& $4 F_{3}+2 F_{7}$ & $2 F_{3}+2 F_{7}$ & $4 / 3$ & & & & \\
& $4 F_{6}$ & $2 F_{6}$ & $2 / 7$ & & & & \\
& $2 F_{7}$ & $2 F_{7}$ & 1 & & & & \\
& $2 F_{7}+3 F_{8}$ & $2 F_{7}+2 F_{8}$ & $8 / 7$ & & & & \\
& $3 F_{8}$ & $2 F_{8}$ & $1 / 2$ & & & & \\
\hline
\end{tabular}

$l=8 E^{3}$ by (5.1). This value is $8 / 7$ when $J=\{(7,3)\}$ and $8 / 15$ when $J=$ $\{(3,1),(5,2)\}$. Therefore Theorem 1.8(i) is deduced.

Remark 5.2. In fact, $P$ is $\mathrm{c} E_{7}$ when $J=\{(7,3)\}$. By the above list, it suffices to exclude the case where $P \in S_{X}$ is $E_{8}$ and $Z=2 F_{7}+3 F_{8}$. In this case, $S \cap E$ defines the 1-cycle $F_{7}+F_{8}$ on $S$. Thus $\left(E \cdot F_{7}\right)$ and $\left(E \cdot F_{8}\right)$ must be $-1 / 7$. On the other hand, using the dual graph for the minimal resolution, we obtain $\left(\left.E\right|_{S} \cdot F_{7}\right)=\left(F_{7}+F_{8} \cdot F_{7}\right)_{S}=-3 / 7$ and $\left(\left.E\right|_{S} \cdot F_{8}\right)=\left(F_{7}+F_{8} \cdot F_{8}\right)_{S}=1 / 7$. It is a contradiction.

Example 5.3. There exist examples of type I. The weighted blow-up of $o \in\left(x_{1}^{2}+\right.$ $\left.x_{2}^{3}+x_{2} x_{3}^{3}+x_{4}^{7}=0\right) \subset \mathbb{C}^{4}$ with weights wt $\left(x_{1}, x_{2}, x_{3}, x_{4}\right)=(7,5,3,2)$ is an example of type I whose $P$ is $\mathrm{c} E_{7}$. The weighted blow-up of $o \in\left(x_{1}^{2}+x_{2}^{3}+x_{3}^{5}+x_{4}^{7}=0\right) \subset \mathbb{C}^{4}$ with weights $\operatorname{wt}\left(x_{1}, x_{2}, x_{3}, x_{4}\right)=(7,5,3,2)$ is an example of type I whose $P$ is $\mathrm{c} E_{8}$. 
When $f$ is of type II or III, by Theorems 4.2 and 4.4 and Remark 1.5, we obtain the possible dual graphs for the partial resolution $S \rightarrow S_{X}$ as follows:

(i) (type IIa or $\mathrm{IIb}^{\vee}$ ) The dual graph is

$$
\text { - }-*\left\{\begin{array}{c}
-\circ \\
1 \\
\vdots \\
-\circ \\
1
\end{array}\right.
$$

where $\bullet$ denotes $\left.H\right|_{S}=D$, the attached numbers are the coefficients in $\left.E\right|_{S}=Z$, and $*$ means that a Du Val singularity of type

(a) $A_{r-1}$ if $f$ is of type IIa,

(b) $A_{2 r-1}$ if $f$ is of type $\mathrm{IIb}^{\vee}$ with $J=\{(r, 1),(r, 1)\}, r \geq 5$,

(c) $A_{5}$ or $E_{6}$ if $f$ is of type $\mathrm{IIb}^{\vee}$ with $J=\{(3,1),(3,1)\}$,

(d) $D_{5}$ if $f$ is of type $\operatorname{IIb}^{\vee}$ with $J=\{(2,1),(4,1)\}$,

appears there respectively. The number of the exceptional curves $\circ$ is $\leq 4$ in type IIa and $\leq a$ in type IIb ${ }^{\vee}$. This bound comes from the value of $(H \cdot[S \cap E])=a E^{3}$.

(ii) (type IIb ${ }^{\vee \vee}$ or III) Set $r_{1}=1, r_{2}=r$ if $f$ is of type III. $P \in S_{X}$ is $A_{n}$ because $\left.H\right|_{S}$ intersects $\left.E\right|_{S}$ at two points. The dual graph is

$$
\bullet-*-\underset{1}{\circ}-\star-\bullet \text { or } \bullet-*-\underset{1}{\circ}-\underset{1}{\circ}-\star-\bullet,
$$

where $\bullet$ denotes $\left.H\right|_{S}=D$, the attached numbers are the coefficients in $\left.E\right|_{S}=Z$, and $*$ and $\star$ mean that a Du Val singularity of type $A_{r_{1}-1}$, respectively $A_{r_{2}-1}$, appears there. Since all the components of $(S \cap E)_{\text {red }}$ are numerically proportional, the number of the exceptional curves $\circ$ is $\leq 2$.

The following table shows all the possibilities of the partial resolution $S \rightarrow S_{X}$ up to permutation in terms of $F_{i}$ :

\begin{tabular}{l|l|l|l}
\hline \multicolumn{1}{c|}{ type of $f$} & \multicolumn{1}{c|}{$S_{X}$} & \multicolumn{1}{c}{$Z$} & \multicolumn{1}{c}{$l$} \\
\hline IIa & $D_{r}$ & $F_{r}$ & $4 / r$ \\
& $D_{r+1}$ & $F_{r}+F_{r+1}$ & $4 / r$ \\
& & $F_{1}+F_{r+1}$ & $1+1 / r$ \\
IIb $^{\vee}, J=\{(r, 1),(r, 1)\}$ & $D_{r+2}$ & $F_{1}+F_{r+1}+F_{r+2}$ & $1+1 / r$ \\
& $D_{2 r}$ & $F_{2 r}$ & $2 / r$ \\
& $D_{2 r+1}$ & $F_{2 r}+F_{2 r+1}$ & $2 / r$ \\
IIb $^{\vee}, J=\{(3,1),(3,1)\}$ & & $F_{1}+F_{2 r+1}$ & $1+1 / 2 r$ \\
IIb $^{\vee}, J=\{(2,1),(4,1)\}$ & $D_{6}$ & $F_{6}$ & $2 / 3$ \\
IIb $^{\vee \vee}$ & $E_{6}$ & $F_{2}$ & 1 \\
& $A_{r_{1}+r_{2}-1}$ & $F_{r_{1}}$ & $3 / 4$ \\
III & $A_{r_{1}+r_{2}}$ & $F_{r_{1}}+F_{r_{1}+1}$ & $1 / r_{1}+1 / r_{2}$ \\
& $A_{r}$ & $F_{r}$ & $1 / r_{1}+1 / r_{2}$ \\
& $A_{r+1}$ & $F_{r}+F_{r+1}$ & $1+1 / r$ \\
\hline
\end{tabular}

We have $l=a E^{3}$ by (5.1). Combining it with Theorem [1.4, [7] and 8], we obtain Theorem [1.8(ii) except the case where $S_{X}$ is $A_{r_{1}+r_{2}}$ in type IIb ${ }^{\vee \vee}$ or $A_{r+1}$ in type III. The remaining case is excluded by Theorem [1.13, proved in Section 6. 
Example 5.4. Let $f$ be the weighted blow-up of $o \in\left(x_{1}^{2}+x_{2}^{2} x_{3}+x_{3}^{2 r}+x_{4}^{r}=0\right) \subset \mathbb{C}^{4}$ with weights $\operatorname{wt}\left(x_{1}, x_{2}, x_{3}, x_{4}\right)=(r, r, 1,2)$, where $r \geq 3$ is an odd integer. $P$ is $\mathrm{c} D_{r+1}$ and $f$ is of type $\operatorname{IIb}^{\vee}$ with $J=\{(r, 1),(r, 1)\}$. The exceptional locus of the partial resolution $S \rightarrow S_{X}$ is reducible.

Example 5.5. Let $f$ be the weighted blow-up of $o \in\left(x_{1}^{2}+x_{2}^{2} x_{3}+x_{3}^{3}+x_{4}^{3}=0\right) \subset \mathbb{C}^{4}$ with weights $\operatorname{wt}\left(x_{1}, x_{2}, x_{3}, x_{4}\right)=(3,1,4,2) . P$ is $\mathrm{c} D_{4}$ and $f$ is of type $\mathrm{IIb}^{\vee}$ with $J=\{(2,1),(4,1)\}$.

\section{Divisorial contractions to $\mathrm{c} A_{n}$ POINTS}

We start with a general method to determine $f$ :

Lemma 6.1. Let $f:(Y \supset E) \rightarrow(X \ni P)$ be a germ of a three-fold divisorial contraction to a Gorenstein point $P$, and set $K_{Y}=f^{*} K_{X}+a E ; X$ is identified with a hypersurface in $\bar{X}:=\mathbb{C}_{x_{1} x_{2} x_{3} x_{4}}^{4}$ :

$$
P \in X \cong o \in(\phi=0) \subset \bar{X}=\mathbb{C}_{x_{1} x_{2} x_{3} x_{4}}^{4} .
$$

Write $m_{i}$ for the multiplicity of $\operatorname{div} x_{i}$ along $E$, that is, the largest integer such that $x_{i} \in f_{*} \mathcal{O}_{Y}\left(-m_{i} E\right)$. We assume that $m_{1}, m_{2}, m_{3}, m_{4}$ have no common factors. Let $d$ be the weighted order of $\phi$ with respect to weights $\operatorname{wt}\left(x_{1}, x_{2}, x_{3}, x_{4}\right)=$ $\left(m_{1}, m_{2}, m_{3}, m_{4}\right)$, and decompose $\phi$ as

$$
\phi=\phi_{d}\left(x_{1}, x_{2}, x_{3}, x_{4}\right)+\phi_{>d}\left(x_{1}, x_{2}, x_{3}, x_{4}\right),
$$

where $\phi_{d}$ is the weighted homogeneous part of weight $d$ and $\phi_{>d}$ the part of weight $>d$. Set $c:=m_{1}+m_{2}+m_{3}+m_{4}-1-d$. Let $\bar{g}:(\bar{Z} \supset \bar{F}) \rightarrow(\bar{X} \ni P)$ be the weighted blow-up of $\bar{X}$ with weights $\mathrm{wt}\left(x_{1}, x_{2}, x_{3}, x_{4}\right)=\left(m_{1}, m_{2}, m_{3}, m_{4}\right), \bar{F}$ its exceptional divisor, and $\bar{D}_{i}$ the birational transform on $\bar{Z}$ of the hyperplane $x_{i}=0$ in $\bar{X}$. Let $Z$ be the birational transform of $X$ on $\bar{Z}$, and $g: Z \rightarrow X$ the induced morphism. Assume that

(i) $\bar{F} \cap Z$ defines an irreducible reduced 2 -cycle $F$ on $Z$, and $F \nsubseteq \operatorname{Sing} Z$,

(ii) $\operatorname{dim}(\operatorname{Sing} \bar{Z} \cap Z) \leq 1$,

(iii) $\operatorname{Sing} Z \subseteq \bigcup_{1 \leq i \leq 4} \bar{D}_{i}$, and

(iv) $F \nsubseteq \bigcup_{1 \leq i \leq 4} \bar{D}_{i}$.

Then $c \leq a$, and the equality holds if and only if $f \cong g$ over $X$.

Proof. $Z$ is $\mathrm{R}_{1}$ from (i), and is Cohen-Macaulay since $Z \subset \bar{Z}$ is locally a cyclic quotient of a hypersurface in $\mathbb{C}^{4}$. Thus $Z$ is normal. By (ii) we can use the adjunction formula and have $K_{Z}=\left.\left(K_{\bar{Z}}+Z\right)\right|_{Z}=g^{*} K_{X}+c F$. Note that $-F$ is $f$ ample and $\mathbb{Q}$-Cartier. Consider the centre on $Z$ of the valuation corresponding to $E$. The multiplicity of $\operatorname{div} x_{i}$ along $E$ equals to that along $F$. Thus, by $\bigcap_{1 \leq i \leq 4} \bar{D}_{i}=\emptyset$ and (iv), this centre is not contained in $\bigcup_{1 \leq i \leq 4} \bar{D}_{i}$, whence it intersects the smooth locus of $Z$ by (iii). Thus the discrepancy $a$ of $E$ with respect to $K_{X}$ is $\geq c$, that of $F$, and the equality holds if and only if $f \cong g$ over $X$ by [7 Lemma 3.4].

Let $f:(Y \supset E) \rightarrow(X \ni P)$ be a germ of a divisorial contraction to a $\mathrm{c} A_{n}$ point $P$ for some $n \geq 2$. $X$ is identified with a hypersurface $\phi=0$ in $\mathbb{C}_{x_{1} x_{2} x_{3} x_{4}}^{4}$ as follows:

$$
P \in X \cong o \in\left(\phi=x_{1} x_{2}+g\left(x_{3}, x_{4}\right)=0\right) \subset \mathbb{C}_{x_{1} x_{2} x_{3} x_{4}}^{4},
$$


where $g$ has total order $n+1$ in $x_{3}, x_{4}$. From Theorems 1.4 and 1.8 ii), $f$ is of type O, II or III. Our goal in this section is to prove Theorem 1.13, the classification of these contractions.

Write $m_{i}$ for the multiplicity of $\operatorname{div} x_{i}$ along $E$. By coordinate change in which $x_{4} \mapsto x_{4}+x_{i}$ for $i=1,2$ or 3 if necessary, we may assume that $m_{4}=1$. By coordinate change $x_{3} \mapsto x_{3}+h\left(x_{4}\right)$ if necessary, we may also assume that $x_{3}+$ $h\left(x_{4}\right) \notin f_{*} \mathcal{O}_{Y}\left(-\left(m_{3}+1\right) E\right)$ for any $h$. Decompose $\phi$ as

$$
\phi=\phi_{\leq m_{1}+m_{2}}\left(x_{1}, x_{2}, x_{3}, x_{4}\right)+\phi_{>m_{1}+m_{2}}\left(x_{1}, x_{2}, x_{3}, x_{4}\right),
$$

where $\phi_{\leq m_{1}+m_{2}}$ is the part of weight $\leq m_{1}+m_{2}$ and $\phi_{>m_{1}+m_{2}}$ is the part of weight $>m_{1}+m_{2}$ with respect to weights wt $\left(x_{1}, x_{2}, x_{3}, x_{4}\right)=\left(m_{1}, m_{2}, m_{3}, 1\right)$. We focus on $\phi_{\leq m_{1}+m_{2}}=x_{1} x_{2}+h\left(x_{3}, x_{4}\right)$. Remark that $h \neq 0$. The homogeneous part $h_{0}$ in $h$ of the minimal weight $d_{0}$ with respect to weights $\operatorname{wt}\left(x_{3}, x_{4}\right)=\left(m_{3}, 1\right)$ always decomposes into a product of $x_{4}^{d_{0}-m_{3}\left\lfloor\frac{d_{0}}{m_{3}}\right\rfloor}$ and $\left\lfloor\frac{d_{0}}{m_{3}}\right\rfloor$ linear combinations of $x_{3}$ and $x_{4}^{m_{3}}$. Hence $h_{0} \notin f_{*} \mathcal{O}_{Y}\left(-\left(d_{0}+1\right) E\right)$ because of the assumption $x_{3}+h\left(x_{4}\right) \notin$ $f_{*} \mathcal{O}_{Y}\left(-\left(m_{3}+1\right) E\right)$ for any $h$. Therefore, $d_{0}$ must be $m_{1}+m_{2}$. We can see that this choice of coordinates satisfies the assumptions in Lemma 6.1, whence

$$
a \geq m_{1}+m_{2}+m_{3}+1-1-\left(m_{1}+m_{2}\right)=m_{3}
$$

and the equality holds if and only if $f \cong g$ in Lemma 6.1 over $X$.

In particular, we have the following in type $\mathrm{O}$ :

Lemma 6.2. If $f$ is of type $O$, then $f$ is the weighted blow-up of $X$ with weights $\operatorname{wt}\left(x_{1}, x_{2}, x_{3}, x_{4}\right)=(i, n+1-i, 1,1)$ for some $1 \leq i \leq n$ in (6.1).

Lemma 6.3. $f$ is not of type IIa with $a=4$.

Proof. If $f$ is of type IIa with $a=4$, then $J=\{(5,2)\}$ from Theorem 3.5, whence $Y$ has exactly one non-Gorenstein point and it is a quotient singularity of type $\frac{1}{5}(1,-1,3)$. Consider a birational morphism $h: Z \rightarrow Y$ such that $Z$ has an $h$ exceptional divisor $F$ the discrepancy of which with respect to $K_{X}$ is 1 . We can choose $n$ different $F$, as valuations, corresponding to the exceptional divisors of the weighted blow-ups of $X$ with weights $\mathrm{wt}\left(x_{1}, x_{2}, x_{3}, x_{4}\right)=(i, n+1-i, 1,1)$ for $1 \leq i \leq n$ in (6.1). Write

$$
\begin{aligned}
K_{Z} & =h^{*} K_{Y}+b F+(\text { others }), \\
h^{*} E & =E_{Z}+m F+(\text { others }),
\end{aligned}
$$

where $E_{Z}$ is the birational transform of $E$. Then $K_{Z}=h^{*}\left(f^{*} K_{X}+4 E\right)+b F+$ (others) $=h^{*} f^{*} K_{X}+4 E_{Z}+(b+4 m) F+$ (others), whence $b=m=1 / 5$ since $5 b, 5 m \in \mathbb{Z}$. Hence $F$ is determined uniquely because $Y$ has a quotient singularity of index 5 as the unique non-Gorenstein point. It contradicts that we can choose $n \geq 2$ different $F$.

The case where $f$ is of type IIa with $a=2$, type IIb or III remains. We can see the next lemma by following [8, Claim 6.13] faithfully:

Lemma 6.4. Let $f:(Y \supset E) \rightarrow(X \ni P)$ be a germ of a three-fold divisorial contraction to a singular Gorenstein point $P$, and set $K_{Y}=f^{*} K_{X}+a E$. Assume that $f$ is of type IIb or III, and set $r_{1}=1$ if $f$ is of type III. $P \in X$ is identified as

$$
P \in X \cong o \in(\phi=0) \subset \mathbb{C}_{x_{1} x_{2} x_{3} x_{4}}^{4} .
$$

Assume that the multiplicity of $\operatorname{div} x_{4}$ along $E$ is 1 . Then 
(i) for $1 \leq j \leq \min \left\{r_{1}, a\right\}$,

$$
f_{*} \mathcal{O}_{Y}(-j E)=\left(x_{1 j}, x_{2 j}, x_{3 j}, x_{4}^{i}\right) \mathcal{O}_{X}
$$

for some $x_{i j}=x_{i}+p_{i j}\left(x_{4}\right)(i=1,2,3)$, where

$$
p_{i j} \in \bigoplus_{k=1}^{j-1} \mathbb{C} x_{4}^{k} \subset \mathbb{C}\left[x_{4}\right] \text {. }
$$

(ii) Assume that $r_{1}<a$.

(a) The linear map

$$
\bigoplus_{i=1}^{3} \mathbb{C} x_{i r_{1}} \rightarrow f_{*} \mathcal{O}_{Y}\left(-r_{1} E\right) /\left(f_{*} \mathcal{O}_{Y}\left(-\left(r_{1}+1\right) E\right)+\left(x_{4}^{r_{1}}\right) \mathcal{O}_{X}\right)
$$

has kernel of dimension 2 .

(b) We assume that $x_{3 r_{1}} \notin f_{*} \mathcal{O}_{Y}\left(-\left(r_{1}+1\right) E\right)+\left(x_{4}^{r_{1}}\right) \mathcal{O}_{X}$. In this situation, for $r_{1}<j \leq a$,

$$
\begin{gathered}
f_{*} \mathcal{O}_{Y}(-j E)=\left(x_{1 j}, x_{2 j}\right) \mathcal{O}_{X}+\sum_{(k, l) \in \bigcup_{s \geq j} J_{s}}\left(x_{3 r_{1}}^{k} x_{4}^{l}\right) \mathcal{O}_{X} \\
\text { for some } x_{i j}=x_{i}+p_{i j}\left(x_{3 r_{1}}, x_{4}\right)(i=1,2) \text {, where } \\
p_{i j} \in \bigoplus_{(k, l) \in \bigcup_{1 \leq s<j} J_{s}} \mathbb{C} x_{3 r_{1}}^{k} x_{4}^{l} \subset \mathbb{C}\left[x_{3 r_{1}}, x_{4}\right], \\
J_{s}:=\left\{(k, l) \in \mathbb{Z}_{\geq 0}^{2} \mid r_{1} k+l=s\right\} .
\end{gathered}
$$

Remark 6.5. A similar result holds even if $f$ is of type IIa with $a=2$ by $d(-1)=$ $\chi\left(\mathcal{Q}_{-1}\right)=1$ in Remark 2.5(i). Assume that $f$ is of type IIa with $a=2$ and the multiplicity of $\operatorname{div} x_{4}$ along $E$ is 1 . Then $x_{1}, x_{2}, x_{3} \in f_{*} \mathcal{O}_{Y}(-2 E)+\left(x_{4}^{2}\right) \mathcal{O}_{X}$.

Assume that $f$ is either of type IIa with $a=2$ or of type $\operatorname{IIb}^{\vee}$ with $J=$ $\{(r, 1),(r, 1)\}$. Then $m_{3} \geq a$ by Lemma 6.4 and Remark 6.5 Assume that $f$ is either of type IIb ${ }^{\vee \vee}$ or of type III, and set $r_{1}=1, r_{2}=r$ if $f$ is of type III. We start with an identification (6.1). Write $m_{i}$ for the multiplicity of $\operatorname{div} x_{i}$ along $E ; m_{4}=1$ is assumed by coordinate change. Because a general element in $f_{*} \mathcal{O}_{Y}(-a E)$ gives a Du Val singularity $P \in S_{X}$ at worst of type $A_{r_{1}+r_{2}}$ by the results in Section 5 this element must be of the form $c x_{3}+\cdots$ with $c \neq 0$ by Lemma 6.4. Hence by coordinate change such that $x_{3} \mapsto x_{3}+h\left(x_{1}, x_{4}\right)$ or $x_{3}+h\left(x_{2}, x_{4}\right)$ if necessary, we may assume that $x_{3} \in f_{*} \mathcal{O}_{Y}(-a E)$ by Lemma 6.4 Therefore $m_{3} \geq a$. Hence we have the following proposition by (6.2):

Lemma 6.6. Assume that $f$ is of type IIb or III. Unless $f$ is of type IIb ${ }^{\vee}$ with $J=\{(2,1),(4,1)\}, f$ is the weighted blow-up of $X$ with weights $\operatorname{wt}\left(x_{1}, x_{2}, x_{3}, x_{4}\right)=$ $\left(m_{1}, m_{2}, a, 1\right)$ with respect to the coordinates in (6.1).

Finally we consider the exceptional case where $f$ is of type $\operatorname{IIb}^{\vee}$ with $J=$ $\{(2,1),(4,1)\}$.

Lemma 6.7. If $f$ is of type II $\vee^{\vee}$ with $J=\{(2,1),(4,1)\}$, then

(i) $P$ is a $c A_{2}$ point isomorphic to $o \in\left(x_{1} x_{2}+x_{3}^{3}+g_{\geq 4}\left(x_{3}, x_{4}\right)=0\right) \subset$ $\mathbb{C}_{x_{1} x_{2} x_{3} x_{4}}^{4}$, where $g_{\geq 4}$ has total order $\geq 4$ in $x_{3}, x_{4}$.

(ii) $Y$ has exactly one non-Gorenstein point $Q$, and it is isomorphic to $o \in$

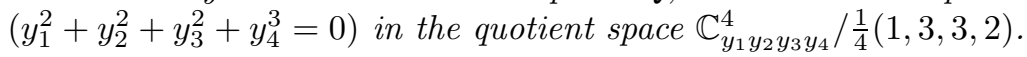


(iii) $K_{Y}=f^{*} K_{X}+3 E$.

Proof. By Remark 1.5 we have (iii) and $Y$ has exactly one non-Gorenstein point $Q$. The germ $Q \in Y$ is described as in Remark 1.5(iii). Because $Y$ has Gorenstein index 4 and $a=3$, for any valuation $v$ with discrepancy 1 with respect to $K_{X}$, the centre of $v$ on $Y$ is $Q$ and $v$ has discrepancy $1 / 4$ with respect to $K_{Y}$, as in the proof of Lemma 6.3. Since there are $n$ such valuations as mentioned in the proof of Lemma 6.3, $Q \in Y$ must have an isomorphism in (iii) by [6, Theorem 7.4] and the description in Remark 1.5 (ii). In this case, there exist exactly two valuations with centre $Q$ the discrepancy of which with respect to $K_{Y}$ is $1 / 4$ by [6, Theorem 7.4]. Thus $P$ has to be $\mathrm{c} A_{2}$.

We take an isomorphism $P \in X \cong o \in\left(x_{1} x_{2}+g_{3}\left(x_{3}, x_{4}\right)+g_{>4}\left(x_{3}, x_{4}\right)=0\right) \subset \mathbb{C}^{4}$, where $g_{3}$ is the part of order 3 and $g_{\geq 4}$ that of order $\geq 4$. To see (ii) we have to show that $g_{3}$ is cubic, but this comes from the result in Section 5 that there exists a hyperplane section of $P \in X$ which is $E_{6}$ at $P$.

Example 6.8. There exists an example of Lemma 6.7. The weighted blow-up of $o \in\left(x_{1}^{2}+x_{2}^{2}+x_{3}^{3}+x_{1} x_{4}^{2}=0\right) \subset \mathbb{C}^{4}$ with weights $w t\left(x_{1}, x_{2}, x_{3}, x_{4}\right)=(4,3,2,1)$ is such an example.

We have Lemmata 6.2, 6.3, 6.6] and 6.7 We can easily check the sufficient condition for $f$ to be a divisorial contraction. Therefore Theorem 1.13 is deduced.

\section{ACKNOWLEDGEMENTS}

I would like to thank Professor Yujiro Kawamata for his stimulating encouragement. I am grateful to Professor Alessio Corti and Professor Miles Reid for motivating me in this subject. I wrote this paper during my visit to the University of Cambridge, where I enjoyed the warm hospitality of Professor Corti. I am also grateful to my friend Daniel Ryder for improving my English. Financial support was provided by the Japan Society for the Promotion of Science.

\section{REFERENCES}

1. M. Artin, On the solutions of analytic equations, Invent. Math. 5 (1968), 277-291. MR 38:344

2. Algebraic approximation of structures over complete local rings, Publ. Math. Inst. Hautes Étud. Sci. 36 (1969), 23-58. MR 42:3087

3. A. Corti, Singularities of linear systems and 3-fold birational geometry, Explicit birational geometry of 3-folds, Lond. Math. Soc. Lect. Note Ser. 281 (2000), 259-312. MR 2001k:14041

4. A. Corti and M. Mella, Birational geometry of terminal quartic 3-folds I, preprint (2000).

5. S. Cutkosky, Elementary contractions of Gorenstein threefolds, Math. Ann. 280 (1988), 521525. MR 89k:14070

6. T. Hayakawa, Blowing ups of 3-dimensional terminal singularities, Publ. Res. Ins. Math. Sci. 35 (1999), 515-570. MR 2002a:14038

7. M. Kawakita, Divisorial contractions in dimension three which contract divisors to smooth points, Invent. Math. 145 (2001), 105-119. MR 2002h:14025

8. Divisorial contractions in dimension three which contract divisors to compound $A_{1}$ points, Compos. Math. 133 (2002), 95-116.

9. Y. Kawamata, Divisorial contractions to 3-dimensional terminal quotient singularities, Higherdimensional complex varieties, de Gruyter (1996), 241-246. MR 98g:14005

10. Y. Kawamata, K. Matsuda and K. Matsuki, Introduction to the minimal model problem, Adv. Stud. Pure Math. 10 (1987), 283-360. MR 89e:14015

11. J. Kollár and S. Mori, Classification of three-dimensional flips, J. Am. Math. Soc. 5 (1992), 533-703. MR 93i:14015 
12. _ Birational geometry of algebraic varieties, Camb. Tracts Math. 134 (1998). MR 2000b: 14018

13. J. Kollár and N. Shepherd-Barron, Threefolds and deformations of surface singularities, Invent. Math. 91 (1988), 299-338. MR 88m:14022

14. D. Markushevich, Minimal discrepancy for a terminal cDV singularity is 1, J. Math. Sci. Tokyo 3 (1996), 445-456. MR 97k:14018

15. S. Mori, Threefolds whose canonical bundles are not numerically effective, Ann. Math. 116 (1982), 133-176. MR 84e:14032

16. - On 3-dimensional terminal singularities, Nagoya Math. J. 98 (1985), 43-66. MR 86m:14003

17. (1988), 117-253. MR 89a:14048

18. M. Reid, Minimal models of canonical 3-folds, Adv. Stud. Pure Math. 1 (1983), 131-180. MR 86a: 14010

19.

20. $\longrightarrow$, Young person's guide to canonical singularities, Proc. Symp. Pure Math. 46 (1987), 345-414. MR 89b:14016

21. V. Shokurov, Smoothness of a general anticanonical divisor on a Fano variety, Izv. Akad. Nauk SSSR Ser. Mat. 43 (1979), 430-441. MR 80h:14020

22. H. Takagi, On classification of $\mathbb{Q}$-Fano 3-folds of Gorenstein index 2 II, Nagoya Math. J. 167 (2002), 157-216

Graduate School of Mathematical Sciences, University of Tokyo, 3-8-1 Komaba, Meguro, Tokyo 153-8914, JAPAN

E-mail address: kawakita@ms.u-tokyo.ac.jp 